\title{
MONODROMY OF A FAMILY OF HYPERSURFACES CONTAINING A GIVEN SUBVARIETY
}

\author{
ANIA OTWINOWSKA AND MORIHIKO SAITO
}

\begin{abstract}
For a subvariety of a smooth projective variety, consider the family of smooth hypersurfaces of sufficiently large degree containing it, and take the quotient of the middle cohomology of the hypersurfaces by the cohomology of the ambient variety and also by the cycle classes of the irreducible components of the subvariety. Using Deligne's semisimplicity theorem together with Steenbrink's theory for semistable degenerations, we give a simpler proof of the first author's theorem (with a better bound of the degree of hypersurfaces) that this monodromy representation is irreducible.
\end{abstract}

\section{Introduction}

It is well known after Noether and Lefschetz that, for a general smooth hypersurface $X$ in $\mathbb{P}_{\mathbb{C}}^{3}$, the subspace of Hodge cycles in the middle cohomology $H^{2}(X, \mathbb{Q})$ is generated by that of $\mathbb{P}_{\mathbb{C}}^{3}$ (i.e. it is 1-dimensional) if the degree of the hypersurface is at least 4 . This follows from the irreducibility of the monodromy representation on the primitive cohomology of the hypersurfaces. Sometimes we want to consider a family of hypersurfaces containing a given closed subvariety $Z$, and ask if an analogue of the above assertion holds.

More generally, let $Y$ be an irreducible smooth complex projective variety embedded in a projective space, and $Z$ be a (possibly reducible) closed subvariety of $Y$. Let $Z_{\{j\}}=\left\{x \in Z: \operatorname{dim} T_{x} Z=j\right\}$, where $T_{x} Z$ denotes the Zariski tangent space. In this paper we assume

$$
\operatorname{dim} Z_{\{j\}}+j \leq \operatorname{dim} Y-1 \text { for any } j \leq \operatorname{dim} Y .
$$

In particular, $2 \operatorname{dim} Z \leq \operatorname{dim} Y-1$. Let $\mathcal{I}_{Z}$ be the ideal sheaf of $Z$, and $\delta$ be a positive integer such that $\mathcal{I}_{Z}(\delta)\left(:=\mathcal{I}_{Z} \otimes \mathcal{O}_{Y}(\delta)\right)$ is generated by global sections, where $\mathcal{O}_{Y}(i)$ denotes the restriction of $\mathcal{O}(i)$ on the ambient projective space. S. Kleiman and A. Altman [13] then proved that condition (0.1) implies the existence of a smooth hypersurface section $X$ of degree $d$ of $Y$ containing $Z$ for any $d>\delta$ (where their condition for $\delta$ is slightly different from ours). Actually, condition (0.1) is also a necessary condition, see [19] (or (1.4) below).

Let $m=\operatorname{dim} X$, and $H^{m}(X, \mathbb{Q})^{\text {van }}$ denote the orthogonal complement of the injective image of $H^{m}(Y, \mathbb{Q})$ in $H^{m}(X, \mathbb{Q})$, which is called the vanishing part or the vanishing cohomology, because it is generated by the vanishing cycles of a Lefschetz pencil using the Picard-Lefschetz formula. Let $H^{m}(X, \mathbb{Q})_{Z}^{\text {van }}$ denote the subspace of $H^{m}(X, \mathbb{Q})^{\text {van }}$ generated by the cycle classes of the maximal dimensional irreducible components of $Z$ modulo the image of $H^{m}(Y, \mathbb{Q}$ ) (using the orthogonal

Date: Jan. 3, 2004, v.2.1. 
decomposition) if $m=2 \operatorname{dim} Z$, and $H^{m}(X, \mathbb{Q})_{Z}^{\operatorname{van}}=0$ otherwise. Let $H^{m}(X, \mathbb{Q})_{\perp Z}^{\operatorname{van}}$ be its orthogonal complement in $H^{m}(X, \mathbb{Q})^{\text {van }}$. The first author [19] made the following

0.2. Conjecture. Assume $\operatorname{deg} X \geq \delta+1$. Then the monodromy representation on $H^{m}(X, \mathbb{Q})_{\perp Z}^{\operatorname{van}}$ for the family of hypersurface sections $X$ containing $Z$ as above is irreducible.

In the case $Z$ is smooth, an easy proof was given by C. Voisin, see (1.6). In general, using a degeneration argument inspired by [10], [16], the first author proved the following.

0.3. Theorem [19]. There exists a positive real number $C$ such that Conjecture (0.2) holds if $\operatorname{deg} X \geq C(\delta+1)$.

This was used in an essential way for the proof of the main theorem in [20], which implies the Hodge conjecture for a hypersurface section of sufficiently large degree belonging to some open subset of an irreducible component of the NoetherLefschetz locus of low codimension, and whose argument generalizes that of [18]. In the proof of Theorem (0.3), however, an asymptotic argument is used, and $C$ can be quite large. In this paper, we give a simpler proof of the theorem below by using the theory of nearby cycles ([5], [24]) together with Hodge theory ([2], [4], [22], [25], [29]) and some cohomological properties of Lefschetz pencils ([6], [12], [14], [15], [28]).

0.4. Theorem. Let $\delta$ be as above, and $d$ be a positive integer. Assume either $d \geq 2+\delta$ or a general hypersurface section of degree $d-\delta$ of $Y$ has a nontrivial differential form of the highest degree. Then Conjecture (0.2) holds with the assumption replaced by $\operatorname{deg} X=d$.

The first assumption $d \geq \delta+2$ may be replaced with a weaker condition that the 2 -jets at each point are generated by the global sections of $\mathcal{O}_{Y}(d-\delta)$. The second assumption on a differential form is stable by hypersurface sections, and it is satisfied for any $d \geq \delta+1$ if $Y$ has a nontrivial differential form of the highest degree. The proof of Theorem (0.4) uses Deligne's semisimplicity theorem [4] which implies that irreducibility is equivalent to indecomposability. We take a semistable degeneration as in [10], and calculate the graded pieces of the weight filtration on the nearby cycles using [24]. Then we can proceed by induction on $m$ showing the nontriviality of certain extension classes by calculating the cohomology of a Lefschetz pencil or a non Lefschetz fibration. In the non Lefschetz case, we use a special kind of degeneration generalizing a construction in the surface case in [19] (see (2.7) below) if the condition $d \geq \delta+2$ is satisfied, and use Hodge theory (see (3.2), (3.3) below) if the second assumption on a differential form is satisfied. In the Lefschetz case, the argument is rather easy, see (2.3), (2.5) below. (We can generalize Theorem $(0.4)$ by replacing $\mathcal{O}_{Y}(\delta), \mathcal{O}_{Y}(d-\delta)$ with two ample line bundles $\mathcal{L}_{1}, \mathcal{L}_{2}$ satisfying appropriate conditions even if they are linearly independent in the Picard group of $Y$ tensored with $\mathbb{Q}$, see (4.8) below.)

By a standard argument, Theorem (0.4) implies 
0.5. Corollary. Under the assumption of Theorem (0.4), assume further that $m=2 \operatorname{dim} Z$, and the vanishing cohomology of a general hypersurface section of degree $d$ does not have a Hodge structure of type $(m / 2, m / 2)$. Then the Hodge cycles in the middle cohomology of a general hypersurface section $X$ of degree $d$ of $Y$ containing $Z$ are generated by the image of the Hodge cycles on $Y$ together with the cycle classes of the irreducible components of $Z$. In particular, the Hodge conjecture for $X$ is reduced to that for $Y$.

In Sect. 1, we review the theory of hypersurface sections containing a subvariety (this is mainly a reproduction from [19]). In Sect. 2, we prove the nonvanishing of some extension classes using a topological method, and in Sect. 3, we do it using a Hodge-theoretic method. In Sect. 4, we study the graded pieces of the weight filtration on the nearby cycles, and prove Theorem (0.4).

In this paper, a variety means a (not necessarily reduced nor irreducible) separated scheme of finite type over $\mathbb{C}$, and a point of a variety means a closed point. We say that a member of a family parametrized by the points of a variety is general if the point corresponding to the member belongs to some dense open subvariety of the variety.

\section{Hypersurfaces Containing a Subvariety}

In this section, we review some basic facts in the theory of hypersurface sections containing a subvariety, which will be needed in the proof of Theorem (0.4). We mainly reproduce the arguments in [19], Section 1, see also [13]. Our argument works only in the case of characteristic 0 .

1.1. Hypersurface sections. Let $Y$ be an $(m+1)$-dimensional irreducible smooth projective variety embedded in a projective space $\mathcal{P}$. For a positive integer $i$, let $\mathcal{O}_{Y}(i)$ be the restriction to $Y$ of $\mathcal{O}_{\mathcal{P}}(i)$ on the projective space $\mathcal{P}$, and define

$$
\mathcal{A}^{i}=\Gamma\left(Y, \mathcal{O}_{Y}(i)\right) \text {. }
$$

Note that the restriction morphism

$$
\Gamma\left(\mathcal{P}, \mathcal{O}_{\mathcal{P}}(i)\right) \rightarrow \Gamma\left(Y, \mathcal{O}_{Y}(i)\right)
$$

is not surjective in general (e.g. if $Y$ is a hypersurface section of degree $i$ of an abelian variety contained in $\mathcal{P})$. For $P \in \mathcal{A}^{i}$, we denote by $X_{P}$ the associated hypersurface section of degree $i$ (in a generalized sense unless $P$ belongs to the image of the above morphism). Let

$$
\mathcal{V}^{i}=\left\{P \in \mathcal{A}^{i} \backslash\{0\}: X_{P} \text { is smooth }\right\} .
$$

It is identified with a smooth variety, and $\mathcal{V}^{i} / \mathbb{C}^{*}$ parametrizes the smooth hypersurface sections of $Y$ of degree $i$.

Let $Z$ be a closed subvariety of $Y$, and put

$$
\mathcal{A}_{Z}^{i}=\left\{P \in \mathcal{A}^{i}: Z \subset X_{P}\right\}, \quad \mathcal{V}_{Z}^{i}=\mathcal{A}_{Z}^{i} \cap \mathcal{V}^{i} .
$$

In this section we do not necessarily assume condition (0.1) in Introduction. Let $\delta$ be a positive integer such that $\mathcal{I}_{Z}(\delta)$ is generated by $\mathcal{A}_{Z}^{\delta}$. It is shown by Kleiman 
and Altman [13] that condition (0.1) in Introduction implies the existence of a smooth hypersurface of degree $j$ containing $Z$ for any $j>\delta^{\prime}$, where the definition of $\delta^{\prime}$ in loc. cit. uses the ideal of $Z$ in the projective space $\mathcal{P}$ instead of $\mathcal{I}_{Z}$ (in particular $\delta \leq \delta^{\prime}$, and we may have a strict inequality if the degrees of the defining equations of $Y$ in $\mathcal{P}$ are bigger than those for $Z$ in $Y$ ). More precisely, they showed the following theorem for $d_{i} \geq \delta^{\prime}+1$ and $V_{i}=\mathcal{A}_{Z}^{d_{i}}$, except possibly for the estimate of $\operatorname{dim} \operatorname{Sing} X_{\mathbf{P}}$, which we need later. Concerning the connectedness, we will need it for the proof of Theorem (0.4) only in the cases where $a \geq 0$ and either $r=1$ or 2. Note that the argument is simpler in these cases because $X_{P_{1}}$ is smooth.

In Theorem (1.2) below, we will take vector subspaces $V_{i}$ of $\mathcal{A}_{Z}^{d_{i}}$, and consider the following condition:

$\left(C_{i}\right) d_{i}>\delta$ and there exist a vector subspace $V_{i}^{\prime}$ of $\mathcal{A}_{Z}^{\delta}$ generating $\mathcal{O}_{Y}(\delta)$ outside $Z$ and a vector subspace $V_{i}^{\prime \prime}$ of $\mathcal{A}^{d_{i}-\delta}$ giving an embedding of $Y$ into a projective space such that $V_{i}$ is the image of $V_{i}^{\prime} \otimes V_{i}^{\prime \prime}$.

1.2. Theorem ([13], [19]). Let $a=\operatorname{dim} Y-1-\max \left\{\operatorname{dim} Z_{\{j\}}+j\right\}$ so that condition (0.1) is satisfied if and only if $a \geq 0$. Let $d_{1} \leq \cdots \leq d_{r}$ be integers with $r \leq$ $c_{Z}:=\operatorname{codim}_{Y} Z$. Assume $d_{i} \geq \delta$, and let $V_{i}$ be a vector subspace of $\mathcal{A}_{Z}^{d_{i}}$ generating $\mathcal{I}_{Z} \otimes \mathcal{O}_{Y}\left(d_{i}\right)$. Take a general $\mathbf{P}=\left(P_{1}, \ldots, P_{r}\right)$ in $V_{1} \times \cdots \times V_{r}$, and put $X_{\mathbf{P}}=$ $\bigcap_{1<i<r} X_{P_{i}}$. Then $X_{\mathbf{P}}$ is a complete intersection, $\operatorname{dim} \operatorname{Sing} X_{\mathbf{P}} \leq r-a-2$, and $X_{\mathbf{P}} \backslash Z$ is smooth.

Assume furthermore that $\operatorname{dim} X_{\mathbf{P}} \geq 1$, the above condition $\left(C_{i}\right)$ is satisfied for any integer $i$ in $\left[c_{Z}, r\right]$ in the case where $\operatorname{dim} Y \leq 2 \operatorname{dim} Z+a+2$ or $Z$ is reduced on the complement of a closed subvariety of dimension $<\operatorname{dim} Z$, and $\left(C_{i}\right)$ is satisfied for any integer $i$ in $\left[c_{Z}-1, r\right]$ otherwise. Then $X_{\mathbf{P}} \backslash Z$ is connected. Here $\left[c_{Z}, r\right]=\emptyset$ if $r<c_{Z}$.

Proof. Let $\Pi_{r}=V_{1} \times \cdots \times V_{r}$, and

$$
\Sigma_{r}=\left\{(\mathbf{P}, x) \in \Pi_{r} \times Z: \operatorname{rank}\left(d P_{1}, \ldots, d P_{r}\right)_{x}<r\right\}
$$

where the $d P_{i}$ are defined by taking a local trivialization of $\mathcal{O}(1)$, and the rank is independent of the trivialization because the $X_{P_{i}}$ contain $Z$. We have

$$
T_{x}^{*} Z=m_{Y, x} /\left(\mathcal{I}_{Z, x}+m_{Y, x}^{2}\right),
$$

(where $m_{Y, x}$ denotes the maximal ideal), and the $P \in V_{i}$ generate $\left(\mathcal{I}_{Z, x}+m_{Y, x}^{2}\right) / m_{Y, x}^{2}$ for any $i$ by the definition of $V_{i}$ (taking a local trivialization of $\mathcal{O}(1)$ ). Let

$$
\Sigma_{r, x}=\Sigma_{r} \cap\left(\Pi_{r} \times\{x\}\right) .
$$

By considering the fiber of the projection $\Pi_{r} \rightarrow \Pi_{r-1}$ and by induction on $r$ we get for any $x \in Z_{\{\operatorname{dim} Y-j\}}$,

$$
\operatorname{codim}_{\Pi_{r}} \Sigma_{r, x}=\max (j-r+1,0) \geq j-r+1 .
$$

By definition of $a$ we have $\operatorname{codim}_{Y} Z_{\{\operatorname{dim} Y-j\}} \geq \operatorname{dim} Y-j+a+1$, hence

$$
\operatorname{codim}_{\Pi_{r} \times Y} \Sigma_{r} \geq \operatorname{dim} Y-r+a+2 .
$$


For a general $\mathbf{P} \in \Pi_{r}$, this implies

$$
\operatorname{codim}_{Y} \Sigma_{r} \cap(\{\mathbf{P}\} \times Z) \geq \operatorname{dim} Y-r+a+2 .
$$

So it remains to show the assertion on the smoothness and the connectedness.

For the smoothness, we see that a subvariety of $(Y \backslash Z) \times \Pi_{r}$ defined by the relation $x \in X_{\mathbf{P}}$ for $(x, \mathbf{P}) \in(Y \backslash Z) \times \Pi_{r}$ is smooth. (Indeed, in the case $r=1$, the variety is defined by $\sum_{i} t_{i} P_{i}$ where $\left(P_{i}\right)$ is a basis of $V_{1}$ as a vector space, and $\left(t_{i}\right)$ is the corresponding coordinate system of $V_{1}$. Furthermore, for any $x \in Y \backslash Z$, some $P_{i}$ does not vanish on a neighborhood of $x$, where the above equation can be divided by this $P_{i}$. The argument is similar for $r>1$.) Then the smoothness follows from the Bertini theorem in characteristic 0 .

For the connectedness, we proceed by induction on $r$. Let $Y^{\prime}=X_{\mathbf{P}^{\prime}}$ for a general $\mathbf{P}^{\prime} \in \Pi_{r-1}$, where $Y^{\prime}=Y$ if $r=1$. Set $b=0$ if $\operatorname{dim} Y \leq 2 \operatorname{dim} Z+a+2$ or $Z$ is reduced on the complement of a closed subvariety of $\operatorname{dimension}<\operatorname{dim} Z$, and $b=1$ otherwise. Assume first $r<\operatorname{codim}_{Y} Z-b$. Since $Y^{\prime} \backslash X_{\mathbf{P}}$ is a smooth affine variety of dimension $\geq 2$, its first cohomology with compact supports vanishes by the weak Lefschetz theorem together with Poincaré duality. So $X_{\mathbf{P}}$ is connected. By Hartshorne's connectedness theorem (see (1.3) below) the connectedness of $X_{\mathbf{P}} \backslash Z$ is then reduced to that $\operatorname{codim}_{X_{\mathbf{P}}} \operatorname{Sing} X_{\mathbf{P}}>1$.

Since Sing $X_{\mathbf{P}} \subset Z$, the last condition is trivially satisfied in the case $r<$ $\operatorname{codim}_{Y} Z-1$. If $r=\operatorname{codim}_{Y} Z-1$ and $Z$ is generically reduced as above so that $b=0$, then $X_{\mathbf{P}}$ is smooth at a general point of $Z$ because each $V_{i}$ generates $\mathcal{I}_{Z} \otimes \mathcal{O}_{Y}\left(d_{i}\right)$. Thus the assertion is proved. Similarly, if $r=\operatorname{codim}_{Y} Z-1$ and $\operatorname{dim} Y \leq 2 \operatorname{dim} Z+a+2$, then $\operatorname{dim} \operatorname{Sing} X_{\mathbf{P}} \leq r-a-2<\operatorname{dim} Z$ and the assertion follows.

Assume now $r \geq \operatorname{codim}_{Y} Z-b$. Consider a rational map of $Y^{\prime}$ to a projective space defined by the restriction of the linear system $V_{r}$ to $Y^{\prime}$. It induces an embedding of $Y^{\prime} \backslash Z$ because $\left.V_{r}^{\prime}\right|_{Y^{\prime}}$ generates $\mathcal{O}_{Y^{\prime}}(\delta)$ outside $Z$ and $\left.V_{r}^{\prime \prime}\right|_{Y^{\prime}}$ induces an embedding. So $X_{\mathbf{P}} \backslash Z$ is isomorphic to a general hyperplane section of $Y^{\prime} \backslash Z$ for this embedding. Since $Y^{\prime} \backslash Z$ is a smooth connected variety of dimension at least 2 , the Bertini theorem in characteristic 0 implies that $X_{\mathbf{P}} \backslash Z$ is connected. This completes the proof of Theorem (1.2).

1.3. Complement to the proof of Theorem (1.2). A local complete intersection is irreducible if it is connected and the singular locus has codimension $>1$. This follows from Hartshorne's connectedness theorem (see e.g. [9], Th. 18.12), because a local complete intersection is Cohen-Macaulay. It also follows from the theory of perverse sheaves [1], considering the long exact sequence of perverse cohomology sheaves associated to the distinguished triangle $\mathbb{Q}_{X_{0}} \rightarrow \mathbb{Q}_{X_{1}} \oplus \mathbb{Q}_{X_{2}} \rightarrow \mathbb{Q}_{X_{3}} \rightarrow$ where $X_{0}=X_{1} \cup X_{2}, X_{3}=X_{1} \cap X_{2}$. Indeed, if $X_{0}$ is a local complete intersection of dimension $n$ and $X_{3}$ has dimension $\leq n-2$, then $\mathbb{Q}_{X_{0}}[n]$ is a perverse sheaf (i.e. ${ }^{p} \mathcal{H}^{n} \mathbb{Q}_{X_{0}}=\mathbb{Q}_{X_{0}}[n]$, see e.g. [7], Th. 5.1.19.) and ${ }^{p} \mathcal{H}^{j} \mathbb{Q}_{X_{3}}=0$ for $j \geq n-1$, see [1]. So $\mathbb{Q}_{X_{0}}[n]$ is the direct sum of ${ }^{p} \mathcal{H}^{n} \mathbb{Q}_{X_{i}}$ for $i=1,2$, and it is a contradiction if $X_{0}$ is connected. 
The following gives a converse of [13] (and we reproduce the arguments here for the convenience of the reader).

1.4. Theorem [19]. Assume $d \geq \delta+1$. If there exists a smooth hypersurface section of degree d containing $Z$, then condition (0.1) in Introduction is satisfied.

Proof. Let $U$ be a non empty smooth open subvariety of $Z_{\{\operatorname{dim} Y-j\}}$ such that $\operatorname{dim} U=\operatorname{dim} Z_{\{\operatorname{dim} Y-j\}}$ and $\mathcal{O}(1)$ is trivialized over $U$. Let

$$
\mathcal{E}=\operatorname{Ker}\left(\left.T^{*} Y\right|_{U} \rightarrow \bigcup_{x \in U} T_{x}^{*} Z\right)
$$

It is a vector bundle of rank $j$ over $U$, and the associated coherent sheaf $\mathcal{O}_{U}(\mathcal{E})$ is generated by $d P$ for $P \in \mathcal{A}_{Z}^{\delta}$ (where we fix a trivialization of $\mathcal{O}(1)$ over $U$ ). So we may assume that $\mathcal{E}$ is trivialized by $P_{1}, \ldots, P_{j} \in \mathcal{A}_{Z}^{\delta}$.

Since $d \geq \delta+1$, we see that the $d P$ for $P \in \mathcal{A}_{Z}^{d}$ generate the 1 -jets of $\mathcal{O}_{U}(\mathcal{E})$, i.e. they generate $\mathcal{O}_{U}(\mathcal{E})_{x} \otimes \mathcal{O}_{U, x} / m_{U, x}^{2}$ over $\mathbb{C}$ for any $x \in U$ (because there are smooth hypersurface sections of degree $d-\delta$ which give local coordinates of $U$ at $x)$.

If the assumption of (1.4) is satisfied, then $d P$ for a general $P \in \mathcal{V}_{Z}^{d}$ gives a nowhere vanishing section of $\mathcal{E}$ over $U$ because $X_{P}$ is smooth. Since condition (0.1) is equivalent to $\operatorname{dim} Z_{\{\operatorname{dim} Y-j\}}<j$ for any $j$, the assertion is reduced to the following:

1.5. Lemma. Let $X$ be a smooth variety of dimension $\geq j$, and $\mathcal{E}$ be a trivial vector bundle of rank $j$ over $X$. Let $V$ be a finite dimensional vector subspace of $\Gamma(X, \mathcal{E})$ which generates the 1 -jets at every point of $X$. Then for a general $\sigma \in V$, the zero locus $\sigma^{-1}(0) \subset X$ is non empty.

Proof. Since $\mathcal{E}$ is trivial, we may identify $\sigma \in \Gamma(X, \mathcal{E})$ with a morphism of $X$ to a vector space $E$. We have a natural morphism $\theta: V \times X \rightarrow E$ which sends $(\sigma, x)$ to $\sigma(x)$. Let $Y=\theta^{-1}(0)$. Then it is enough to show that $Y$ is dominant over $V$.

Since $V$ generates the 1-jets and $\operatorname{dim} X \geq j$, there exists $y=(\sigma, x) \in Y$ such that the differential $d_{x} \sigma: T_{x} X \rightarrow T_{\sigma(x)} E=E$ of $\sigma: X \rightarrow E$ (by the above identification) is surjective. Consider the commutative diagram

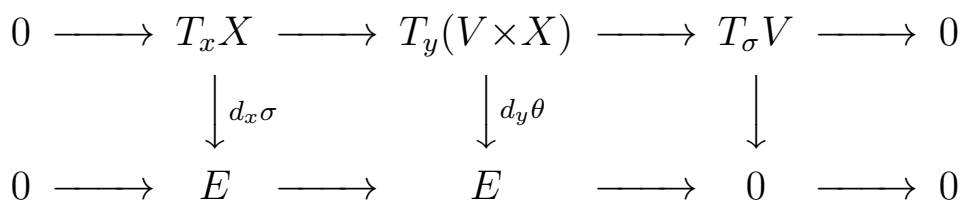

where the top row is induced by the inclusion $\{\sigma\} \times X \rightarrow V \times X$ and the projection $V \times X \rightarrow V$. Then the surjectivity of $d_{x} \sigma$ implies that of $\operatorname{Ker} d_{y} \theta \rightarrow T_{\sigma} V$ by the snake lemma. So the assertion follows because $\operatorname{Ker} d_{y} \theta=T_{y} Y$. This completes the proof of Theorem (1.4).

1.6. Voisin's proof of Conjecture (0.2) in the smooth case. Consider a rational morphism of $Y$ to a projective space $\mathcal{P}$ defined by the linear system $\mathcal{A}_{Z}^{\delta+1}$. It induces an embedding of $Y \backslash Z$, in particular, it is birational to the image. Let $\widetilde{Y}$ be the closure of the image. If $Z$ is smooth, we see that $\widetilde{Y}$ is the blow-up of $Y$ along $Z$. For a smooth hyperplane section $\widetilde{Y}_{s}$ of $\mathcal{P}$ and the corresponding smooth 
hypersurface section $Y_{s}$ of $Y$, there is a morphism $H^{m}\left(\tilde{Y}_{s}\right)^{\text {van }} \rightarrow H^{m}\left(Y_{s}\right)^{\text {van }}$ by the functoriality of the Gysin morphisms, and it is injective by the irreducibility of $\left\{H^{m}\left(\widetilde{Y}_{s}\right)^{\text {van }}\right\}$. Calculating the cohomology of the blow-up, we see that the dimension of its cokernel coincides with the number of the $(\mathrm{m} / 2)$-dimensional irreducible components of $Z$. So Conjecture $(0.2)$ is proved in this case.

\section{Topological Method}

In this section, we prove the nonvanishing of certain extension classes when the condition $d \geq \delta+2$ in Theorem (0.4) is satisfied.

2.1. Exact sequences. Let $Y$ be a connected smooth complex algebraic variety, and $X$ be a divisor on $Y$ with the inclusion $i: X \rightarrow Y$. Put $U=Y \backslash X$ with the inclusion $j: U \rightarrow Y$. Let $f: Y \rightarrow S$ be a proper morphism to a smooth variety $S$, and let $g=f \circ i: X \rightarrow S, h=f \circ j: U \rightarrow S$ be the restrictions of $f$. Put $m=\operatorname{dim} Y-\operatorname{dim} S$. We have a long exact sequence of constructible sheaves

$$
\begin{aligned}
\rightarrow R^{m-1} f_{*} \mathbb{Q}_{Y} & \stackrel{\gamma_{m-1}}{\rightarrow} R^{m-1} g_{*} \mathbb{Q}_{X} \\
& \rightarrow R^{m} h_{!} \mathbb{Q}_{U} \rightarrow R^{m} f_{*} \mathbb{Q}_{Y} \stackrel{\gamma_{m}}{\rightarrow} R^{m} g_{*} \mathbb{Q}_{X} \rightarrow,
\end{aligned}
$$

using the distinguished triangle $j_{!} \mathbb{Q}_{U} \rightarrow \mathbb{Q}_{Y} \rightarrow i_{*} \mathbb{Q}_{X} \stackrel{+1}{\rightarrow}$ together with the functor $\mathbf{R} f_{*}=\mathbf{R} f_{!}$. Since $f, g$ are proper, the base change holds so that the stalk of the direct image is isomorphic to the (relative) cohomology of the fiber. Let

$$
\mathcal{F}=R^{m} h_{!} \mathbb{Q}_{U}, \quad \mathcal{F}^{\prime}=\text { Coker } \gamma_{m-1}, \quad \mathcal{F}^{\prime \prime}=\operatorname{Ker} \gamma_{m},
$$

so that we have a short exact sequence of constructible sheaves

$$
0 \rightarrow \mathcal{F}^{\prime} \rightarrow \mathcal{F} \rightarrow \mathcal{F}^{\prime \prime} \rightarrow 0 \text {. }
$$

Let $X_{s}=g^{-1}(s)$, etc. If $f, g$ are smooth projective and $X_{s}$ is a hypersurface section of $Y_{s}$ for $s \in S$, then we have

$$
\mathcal{F}_{s}^{\prime}=H^{m-1}\left(X_{s}, \mathbb{Q}\right)^{\text {van }}, \quad \mathcal{F}_{s}=H^{m}\left(Y_{s}, X_{s}, \mathbb{Q}\right), \quad \mathcal{F}_{s}^{\prime \prime}=H^{m}\left(Y_{s}, \mathbb{Q}\right)^{\text {prim }} .
$$

We will assume that they are nonzero (because otherwise the extension class vanishes).

If $f, g$ are smooth morphisms, then (2.1.1) and (2.1.2) are exact sequences of local systems, which underlie naturally variation of mixed Hodge structures, see [4], [25] (and also [22]). In the application we will also consider the dual of (2.1.2)

$$
0 \rightarrow \mathcal{F}^{\prime \prime *} \rightarrow \mathcal{F}^{*} \rightarrow \mathcal{F}^{*} \rightarrow 0,
$$

where * denotes the dual variation of mixed Hodge structure. Note that

$$
\mathcal{F}^{* *}=\mathcal{F}^{\prime}(m-1), \quad \mathcal{F}^{\prime \prime *}=\mathcal{F}^{\prime \prime}(m), \quad \mathcal{F}_{s}^{*}=H^{m}\left(Y_{s} \backslash X_{s}, \mathbb{Q}\right)(m),
$$

where $(m)$ denotes the Tate twist, see [4].

2.2. Lefschetz pencils. With the above notation, assume $S=\mathbb{P}^{1}$ and $g: X \rightarrow S$ is a Lefschetz pencil of a smooth projective variety $Y_{0}$. Let $H^{m-1}\left(X_{s}, \mathbb{Q}\right)^{\text {van }}$ be the subgroup of $H^{m-1}\left(X_{s}, \mathbb{Q}\right)$ generated by the vanishing cycles, and assume it 
nonzero. Let $s_{0}$ be any point in the discriminant of the Lefschetz pencil. Since the discriminant in the parameter space of hypersurfaces is irreducible, the last assumption is equivalent to the surjectivity of the restriction morphism

$$
H^{m-1}\left(X_{s}, \mathbb{Q}\right) \rightarrow H^{m-1}\left(B_{s}, \mathbb{Q}\right)
$$

for $s$ sufficiently near $s_{0}$, where $B$ is a small ball in $X$ around the unique singular point of $X_{s_{0}}$, and $B_{s}:=B \cap X_{s}$ is called a Milnor fiber, see [17]. This implies that the cospecialization morphism

$$
H^{m}\left(X_{s_{0}}, \mathbb{Q}\right) \rightarrow H^{m}\left(X_{s}, \mathbb{Q}\right)
$$

is an isomorphism, using a long exact sequence. So we get

$$
R^{j} g_{*} \mathbb{Q}_{X} \text { is a constant sheaf on } S \text { for any } j \neq m-1
$$

see [12], where the case $j=m$ follows from the above argument, and the other cases are easy. As a corollary, we get in this case

$$
\mathcal{F}^{\prime \prime} \text { in }(2.1 .2) \text { is a constant sheaf. }
$$

The above argument also implies for $j=m-1$ that $R^{m-1} g_{*} \mathbb{Q}_{X}$ is a (shifted) intersection complex, i.e.

$$
R^{m-1} g_{*} \mathbb{Q}_{X}=j_{*} j^{*} R^{m-1} g_{*} \mathbb{Q}_{X},
$$

where $j: S^{\prime} \rightarrow S$ is the inclusion of a dense open subvariety over which $g$ is smooth. (This also follows from the local invariant cycle theorem [3] or the decomposition theorem [1].)

The following proposition was proved in [19] using a generalization of the PicardLefschetz formula together with an assertion concerning the vanishing cycles of a Lefschetz pencil and related to the classical work of Lefschetz and Poincaré (see [14], [15], [28]). We give here a simple proof of the proposition using the above cohomological property of the Lefschetz pencil.

2.3. Proposition (Lefschetz pencil case) [19]. With the notation and the assumptions of (2.1), assume $S=\mathbb{P}^{1}, Y=Y_{0} \times S, f=\mathrm{pr}_{2}$, and $g: X \rightarrow S$ is a Lefschetz pencil of $Y_{0}$. Let $S^{\prime}$ be any non empty open subvariety of $S$ over which $g$ is smooth. Then for any nonzero local subsystem $\mathcal{G}$ of $\left.\mathcal{F}^{\prime \prime}\right|_{S^{\prime}}$, the composition of the inclusion $\left.\mathcal{G} \rightarrow \mathcal{F}^{\prime \prime}\right|_{S^{\prime}}$ with the extension class defined by the restriction of the short exact sequence (2.1.2) to $S^{\prime}$ is nontrivial as an extension of local systems.

Proof. Since the local system $\left.\mathcal{F}^{\prime \prime}\right|_{S^{\prime}}$ is constant, we may assume that $\mathcal{G}$ has rank 1 , and is generated by $u \in H^{0}\left(S^{\prime},\left.\mathcal{F}^{\prime \prime}\right|_{S^{\prime}}\right)=H^{m}\left(Y_{0}, \mathbb{Q}\right)^{\text {prim }}$. Assume $u$ is the image of $v \in H^{0}\left(S^{\prime},\left.\mathcal{F}\right|_{S^{\prime}}\right)$. Then it gives a section of $(2.1 .2)$ on $\mathcal{G}$. So it is enough to show that $u=0$ in this case.

We see that $\mathcal{F}$ is a (shifted) intersection complex by (2.1.2), because $\mathcal{F}^{\prime}$ and $\mathcal{F}^{\prime \prime}$ are (shifted) intersection complexes with support $S$. So $H^{0}(S, \mathcal{F})=H^{0}\left(S^{\prime},\left.\mathcal{F}\right|_{S^{\prime}}\right)$, and we may replace $S^{\prime}$ with $S$ or any nonempty open subvariety of $S$. Thus we may assume that $S^{\prime}=S \backslash\left\{s_{0}\right\}$ and $X_{s_{0}}$ is smooth. 
Let $h^{\prime}: U^{\prime} \rightarrow S^{\prime}$ be the restriction of $h$ over $S^{\prime}$, where $U^{\prime}=Y^{\prime} \backslash X^{\prime}$. Consider the Leray spectral sequence

$$
E_{2}^{p, q}=H^{p}\left(S^{\prime}, R^{q} h_{!}^{\prime} \mathbb{Q}_{U^{\prime}}\right) \Rightarrow H^{p+q}\left(Y^{\prime}, X^{\prime} ; \mathbb{Q}\right) .
$$

This degenerates at $E_{2}$, because $E_{2}^{p, q}=0$ unless $p=0$ or 1 . Thus we get $w \in$ $H^{m}\left(Y^{\prime}, X^{\prime} ; \mathbb{Q}\right)$ whose image in $H^{0}\left(S^{\prime},\left.\mathcal{F}\right|_{S^{\prime}}\right)$ is $v$. Its image in $H^{0}\left(S^{\prime},\left.\mathcal{F}^{\prime \prime}\right|_{S^{\prime}}\right)=$ $H^{m}\left(Y^{\prime}, \mathbb{Q}\right)=H^{m}\left(Y_{0}, \mathbb{Q}\right)$ is $u$, where the last isomorphism follows from $Y^{\prime}=Y_{0} \times \mathbb{A}^{1}$. Then the image of $u$ in $H^{m}\left(X^{\prime}, \mathbb{Q}\right)$ vanishes. But this is induced by the restriction morphism under the birational morphism $X^{\prime} \rightarrow Y_{0}$. So we can verify that $u$ belongs to the image of the Gysin morphism under the inclusion $X_{s_{0}} \rightarrow Y_{0}$, and we get $u=0$ because $u$ is primitive. This completes the proof of Proposition (2.3).

We also give an outline of the original proof of Proposition (2.3). We start with the explanation of a generalized Picard-Lefschetz formula.

2.4. Generalized Picard-Lefschetz formula. Let $\mathcal{F}$ be a constructible sheaf on a curve $S$ with a local coordinate $t$. Let $\psi_{t} \mathcal{F}, \varphi_{t} \mathcal{F}$ denote the nearby and vanishing cycles, see [5]. Then we have natural morphisms

$$
\text { can }: \psi_{t} \mathcal{F} \rightarrow \varphi_{t} \mathcal{F}, \quad \text { var }: \varphi_{t} \mathcal{F} \rightarrow \psi_{t} \mathcal{F}
$$

such that

$$
T-i d=\text { varocan }: \psi_{t} \mathcal{F} \rightarrow \psi_{t} \mathcal{F},
$$

where $T$ is the monodromy. It is well known that the functors $\psi_{t}, \varphi_{t}$ commute with the functor assigning the dual, and duality exchanges can and var up to a sign, see e.g. [11], [21]. In the case of a Lefschetz pencil, we can identify the morphism can with the restriction to the Milnor fiber (2.2.1).

Assume that $\varphi_{t} \mathcal{F} \simeq \mathbb{Q}$, and can, var are nonzero. Let $\gamma_{0}$ be a generator of $\varphi_{t} \mathcal{F}$, and $\gamma$ be its image in $\psi_{t} \mathcal{F}$ by var. Let $\mathcal{F}^{*}$ be the (shifted) dual of $\mathcal{F}$ which is defined by $\mathbf{R} \mathcal{H} o m\left(\mathcal{F}, \mathbb{Q}_{S}\right)$. Let $\gamma_{0}^{*}$ be the generator of $\varphi_{t} \mathcal{F}^{*}$ such that $\left\langle\gamma_{0}^{*}, \gamma_{0}\right\rangle=1$. Let $\gamma^{*}$ be its image in $\psi_{t} \mathcal{F}^{*}$ by var. Then we have a generalized Picard-Lefschetz formula

$$
T(u)-u= \pm\left\langle\gamma^{*}, u\right\rangle \gamma \quad \text { for } u \in \psi_{t} \mathcal{F}
$$

because $\left\langle\gamma_{0}^{*}, \operatorname{can}(u)\right\rangle= \pm\left\langle\operatorname{var}\left(\gamma_{0}^{*}\right), u\right\rangle$, see also [6]. This was proved in [19] for the cohomology of the complement of a hypersurface section.

2.5. Outline of the original proof of Proposition (2.3) (see [19]). It is enough to show that $\left.\mathcal{F}\right|_{S^{\prime}}$ has no global sections. In this case, the stalk of $\left.\mathcal{F}^{*}\right|_{S^{\prime}}$ is $H_{m}\left(Y_{s}, X_{s}\right)$, and $\gamma^{*}$ can be constructed explicitly using the ball and the Milnor fiber around the critical point (and this coincides with the construction in [14], [15], [28]), because we can identify the morphism can with the restriction to the complement of the Milnor fiber in the ball, see [19]. Furthermore, considering $\gamma^{*}$ at any points of the discriminant of the Lefschetz pencil, they generate $H_{m}\left(Y_{s}, X_{s}\right)$. (This is closely related to the classical work of Lefschetz and Poincaré, and seems to have been known to some people, see [14] and also [15], [28].) So the local system $\left.\mathcal{F}\right|_{S^{\prime}}$ has no global section, and the assertion follows. 
2.6. Non Lefschetz fibration case. Let $Y$ be a connected smooth projective variety embedded in a projective space $\mathcal{P}$, and $X$ be a hypersurface section of $Y$ with at most isolated singularities. We assume $m:=\operatorname{dim} X=\operatorname{dim} Y-1 \geq 1$. Let $Z$ be an irreducible component of $X$ (hence $Z=X$ if $m>1$ ). Let $d$ be an integer $\geq 2$, and $S$ be the parameter space of hypersurfaces of degree $d$ of $\mathcal{P}$ whose intersections $Z_{s}, Y_{s}$ with $Z, Y$ are smooth divisors on $Z \backslash$ Sing $X$ and $Y$ respectively (in particular, the hypersurfaces parametrized by $S$ do not meet $\operatorname{Sing} X$ ).

Let $\left\{H^{m-1}\left(Z_{s}\right)_{Z}^{\operatorname{van}}\right\}_{s \in S}$ be the local subsystem of $\left\{H^{m-1}\left(Z_{s}\right)\right\}_{s \in S}$ generated by the vanishing cycles at general points of the discriminant of the morphism $\bigsqcup_{s \in \bar{S}} Z_{s} \rightarrow$ $\bar{S}$, where $\bar{S}$ is the parameter space of all the hypersurfaces of degree $d$ of $\mathcal{P}$, and $\bigsqcup_{s \in \bar{S}} Z_{s}$ denotes the total space of the associated family of hypersurfaces. If $m>$ 1 and $X=Z$, let $\left\{H^{m-1}\left(Z_{s}\right)^{\text {van }}\right\}_{s \in S}$ be the local subsystem generated by the vanishing cycles for the inclusion $Z_{s} \rightarrow Y_{s}$. By the Picard-Lefschetz formula, the latter is the orthogonal complement of the injective image of the cohomology of $Y_{s}$ (or $Y$ using the weak Lefschetz theorem), and hence contains the former. If $X=Z$ is smooth, they coincide because they are the orthogonal complement of the injective image of $H^{m-1}(Y)$. If $m=1$, let $H^{m-1}\left(Z_{s}\right)^{\mathrm{van}}=H^{m-1}\left(Z_{s}\right)_{Z}^{\operatorname{van}}(=$ $\left.\widetilde{H}^{m-1}\left(Z_{s}\right)\right)$.

Let $S^{\prime}$ be a dense open subvariety of $S$, and $\widetilde{L}$ be any local system on $S^{\prime}$ such that

$$
\left\{H^{m-1}\left(Z_{s}\right)_{Z}^{\operatorname{van}}\right\}_{s \in S^{\prime}} \subset \widetilde{L} \subset\left\{H^{m-1}\left(Z_{s}\right)^{\operatorname{van}}\right\}_{s \in S^{\prime}}
$$

and the restriction of the intersection pairing to $\widetilde{L}$ is nondegenerate. Let $\widetilde{L}^{\perp}$ be the orthogonal complement of $\widetilde{L}$ in $\left\{H^{m-1}\left(Z_{s}\right)\right\}$. Note that the restrictions of the intersection pairing to the injective image of $H^{m-1}(Y)$ and to $H^{m-1}\left(Z_{s}\right)^{\text {van }}$ are non degenerate using Hodge theory (or [1] because it is essentially equivalent to the hard Lefschetz theorem).

Consider the kernel of the composition

$$
H^{m}\left(Y_{s}, Z_{s}\right) \rightarrow H^{m}\left(Y_{s}\right) \rightarrow H^{m+2}(Y)(1),
$$

where the last morphism is the Gysin morphism. Let $H^{m}\left(Y_{s}, Z_{s}\right)_{\widetilde{L}}^{\text {van }}$ be the quotient of the kernel by the image of $\widetilde{L}^{\perp}$.

The following is a generalization of a construction in the surface case in [19], and gives a topological proof of variants of Propositions (3.2) in the non Lefschetz case and (3.3) in the surface case.

2.7. Proposition (Non Lefschetz fibration case). With the above notation and assumptions, we have a short exact sequence of local systems on $S^{\prime}$

$$
0 \rightarrow \widetilde{L} \rightarrow\left\{H^{m}\left(Y_{s}, Z_{s}\right)_{\widetilde{L}}^{\mathrm{van}}\right\} \rightarrow\left\{H^{m}\left(Y_{s}\right)^{\mathrm{van}}\right\} \rightarrow 0
$$

and it does not split if the first and last terms are nonzero.

Proof. The exactness of (2.7.1) is clear by definition. To show the non splitting of (2.7.1), we may assume $S^{\prime}=S$ using the direct image by $S^{\prime} \rightarrow S$. We take a smooth point $O$ of $X$ contained in $Z$, and consider a hypersurface $H_{0}$ in the ambient projective space which intersects $X, Y$ transversely at smooth points outside $O$ 
and whose intersections with $Z, Y$ have an ordinary double point at $O$. (Such a hypersurface exists because the degree of the hypersurface is at least 2.) Then we consider a generic deformation $\left\{H_{\lambda}\right\}$ of $H_{0}$, parametrized by $\Lambda:=\Delta^{m+2}$ where $\Delta$ is a sufficiently small open disk. Using the embedding by $\mathcal{O}_{Y}(d)$ (which changes hypersurfaces into hyperplanes) and an appropriate projection between projective spaces, we may assume locally

$$
\begin{aligned}
Y & =\left\{x_{m+2}=\sum_{1 \leq i \leq m+1} x_{i}^{2}+\Psi\left(x_{1}, \ldots, x_{m+1}\right)\right\} \\
Z & =\left\{x_{m+1}=0\right\} \subset Y, \\
H_{\lambda} & =\left\{x_{m+2}=\sum_{1 \leq i \leq m+1} 2 a_{i} x_{i}+a_{m+2}\right\} \quad \text { for } \lambda=\left(a_{i}\right) \in \Delta^{m+2},
\end{aligned}
$$

in a $(m+2)$-dimensional projective space, where $\left(x_{i}\right)$ is an affine coordinate system and $\Psi\left(x_{1}, \ldots, x_{m+1}\right) \in\left(x_{1}, \ldots, x_{m+1}\right)^{3}$.

Let $Z_{\lambda}=Z \cap H_{\lambda}, Y_{\lambda}=Y \cap H_{\lambda}$. The discriminant of $\left\{Z_{\lambda}\right\}_{\lambda \in \Lambda}$ and $\left\{Y_{\lambda}\right\}_{\lambda \in \Lambda}$ in $\Lambda$ are defined respectively by

$$
\begin{aligned}
& D_{Z}=\left\{a_{m+2}+\sum_{1 \leq i \leq m} a_{i}^{2}+\Phi_{Z}\left(a_{1}, \ldots, a_{m}\right)=0\right\}, \\
& D_{Y}=\left\{a_{m+2}+\sum_{1 \leq i \leq m+1} a_{i}^{2}+\Phi_{Y}\left(a_{1}, \ldots, a_{m+1}\right)=0\right\},
\end{aligned}
$$

where $\Phi_{Z}\left(a_{1}, \ldots, a_{m}\right) \in\left(a_{1}, \ldots, a_{m}\right)^{3}, \Phi_{Y}\left(a_{1}, \ldots, a_{m+1}\right) \in\left(a_{1}, \ldots, a_{m+1}\right)^{3}$.

Take $c \in \Delta \backslash\{0\}$, and put $0^{\prime}=(0) \in \Delta^{m}$. Define

$$
\begin{aligned}
& \Lambda_{c}=\left\{0^{\prime}\right\} \times\{c\} \times \Delta, \quad\{\alpha\}=\Lambda_{c} \cap D_{Z}, \quad\{\beta\}=\Lambda_{c} \cap D_{Y}, \\
& \Lambda_{c}^{\prime}=\Lambda_{c} \backslash\{\alpha, \beta\} .
\end{aligned}
$$

Let $B$ be a sufficiently small open ball around $O$ in the ambient space, and assume $\Delta$ is much smaller. Let

$$
\left.Z_{B}=Z \cap B, \quad Z_{B, \lambda}=Z_{\lambda} \cap B \text { (similarly for } Y\right) .
$$

For $\lambda \in \Lambda_{c} \backslash\{\beta\}$, it is well known that

$$
\begin{aligned}
& \widetilde{H}^{j}\left(Y_{B, \lambda}\right)=\mathbb{Q} \quad \text { for } j=m, \text { and } 0 \text { otherwise, } \\
& \widetilde{H}^{j}\left(Y_{B, \beta}\right)=0 \quad \text { for any } j .
\end{aligned}
$$

and similarly for $Z_{B, \lambda}$ with $\beta$ replaced by $\alpha$, and $m$ by $m-1$.

The local monodromy of $H^{m-1}\left(Z_{B, \lambda}\right)$ (resp. $H^{m}\left(Y_{B, \lambda}\right)$ ) around $\alpha$ (resp. $\beta$ ) is $(-1)^{m}$ (resp. $\left.(-1)^{m+1}\right)$. By $(2.7 .2)$ we have the short exact sequences

$$
\begin{aligned}
& 0 \rightarrow H^{m-1}\left(Z_{B, \lambda}\right) \rightarrow H^{m}\left(Y_{B, \lambda}, Z_{B, \lambda}\right) \rightarrow H^{m}\left(Y_{B, \lambda}\right) \rightarrow 0, \\
& 0 \rightarrow H_{c}^{m-1}\left(Z_{B, \lambda}\right) \rightarrow H_{c}^{m}\left(Y_{B, \lambda} \backslash Z_{B, \lambda}\right) \rightarrow H_{c}^{m}\left(Y_{B, \lambda}\right) \rightarrow 0 .
\end{aligned}
$$

By definition, we have an injective morphism of (2.7.4) to (2.7.1). Taking the dual, we get a surjective morphism of (2.7.1) to (2.7.3) because the intersection form is non degenerate on $\widetilde{L}$. Let $e, e^{\prime}$ denote the extension classes defined by (2.7.3) and (2.7.4) respectively. Then the assertion is reduced to Lemma (2.8) below. Indeed, if $m$ is odd and the composition of $e$ with the surjection

$$
\left\{H^{m}\left(Y_{\lambda}\right)^{\operatorname{van}}\right\}_{\lambda \in \Lambda_{c}^{\prime}} \rightarrow\left\{H^{m}\left(Y_{B, \lambda}\right)\right\}_{\lambda \in \Lambda_{c}^{\prime}}
$$


vanishes, then $\left\{H^{m}\left(Y_{B, \lambda}, Z_{B, \lambda}\right)\right\}$ is isomorphic to a subquotient of $\left\{H^{m}\left(Y_{\lambda}\right)^{\text {van }}\right\}$ (by the nontriviality of $e$ ) and there is a surjection from the kernel of the above morphism to $\left\{H^{m-1}\left(Z_{B, \lambda}\right)\right\}$, but this contradicts the nontriviality of the monodromy of $\left\{H^{m-1}\left(Z_{B, \lambda}\right)\right\}$ around $\alpha$. The argument is similar for $m$ even.

2.8. Lemma. The extension classes e, e defined by (2.7.3), (2.7.4) do not vanish if $m$ is odd or even respectively.

Proof. We first show the non splitting of (2.7.3) for $m$ odd. In this case, the monodromy of $\left\{H^{m}\left(Y_{B, \lambda}, Z_{B, \lambda}\right)\right\}$ around $\beta$ is unipotent, and we have to show that it is not the identity. Let $D \subset \Delta_{c}$ be a sufficiently small open disk with center $\beta$, and restrict the local systems to $D^{*}:=D \backslash\{\beta\}$. The short exact sequence of local systems (2.7.3) on $D^{*}$ is naturally extended to $D$ as constructible sheaves, by using the higher direct images as in (2.1) instead of cohomology.

More precisely, let $Z_{B, D}, Y_{B, D}$ be the restriction of $Z_{B}, Y_{B}$ over $D$. Let $U=$ $Y_{B, D} \backslash Z_{B, D}$ with the inclusion $j_{U}: U \rightarrow Y_{B, D}$ Then the short exact sequence of local systems on $D^{*}$ defined by $(2.7 .3)$ is naturally extended to a short exact sequence of constructible sheaves on $D$

$$
0 \rightarrow \mathcal{G}^{\prime} \rightarrow \mathcal{G} \rightarrow \mathcal{G}^{\prime \prime} \rightarrow 0 \text {. }
$$

This is also defined by the higher direct images of $\left(j_{U}\right) ! \mathbb{Q}_{U}$, etc. under $Y_{B, D} \rightarrow D$. Here the base change holds, because $\left(Y_{\lambda}, Z_{\lambda}\right)$ is transversal to the boundary of $B$. In particular, the stalk at $\beta$ is given by (2.7.3).

Using (2.7.2), we see that $\mathcal{G}^{\prime}=\mathbb{Q}_{D}, \mathcal{G}^{\prime \prime}=\left(j_{D}\right) ! \mathbb{Q}_{D^{*}}$, where $j_{D}: D^{*} \rightarrow D$ is the inclusion morphism. Let $t$ be a local coordinate around $\beta$. Then $\varphi_{t} \mathcal{G}^{\prime}=0$ and $\psi_{t} \mathcal{G}^{\prime}=\psi_{t} \mathcal{G}^{\prime \prime}=\varphi_{t} \mathcal{G}^{\prime \prime}=\mathbb{Q}$ using the bijectivity of can (see (2.4)) for $\mathcal{G}^{\prime \prime}$. The last assertion follows from the distinguished triangle of functors

$$
i_{0}^{*} \rightarrow \psi_{t} \stackrel{\text { can }}{\rightarrow} \varphi_{t} \rightarrow
$$

where $i_{0}:\{\beta\} \rightarrow D$ denotes an inclusion, see [5]. Since $\psi_{t}, \varphi_{t}$ are exact functors, we get $\operatorname{rank} \psi_{t} \mathcal{G}=2, \operatorname{rank} \varphi_{t} \mathcal{G}=1$ together with the surjectivity of can $: \psi_{t} \mathcal{G} \rightarrow \varphi_{t} \mathcal{G}$.

By (2.4.1) the assertion is thus reduced to the injectivity of var $: \varphi_{t} \mathcal{G} \rightarrow \psi_{t} \mathcal{G}$. By duality (see (2.4)) it is equivalent to the surjectivity of can $: \psi_{t} \mathcal{G}^{*} \rightarrow \varphi_{t} \mathcal{G}^{*}$, where $\mathcal{G}^{*}$ is the (shifted) dual of $\mathcal{G}$ which is defined by $\mathbf{R} \mathcal{H o m}\left(\mathcal{G}, \mathbb{Q}_{D}\right)$.

By duality [27] $\mathcal{G}^{*}$ is isomorphic to the derived direct image with compact supports of $\mathbf{R}\left(j_{U}\right)_{*} \mathbb{Q}_{U}$. The stalk at $\beta$ of the higher direct image sheaves is

$$
H_{c}^{j}\left(Y_{B, \beta}, \mathbf{R}\left(j_{\beta}\right)_{*} \mathbb{Q}_{Y_{\beta} \backslash Z_{\beta}}\right),
$$

(where $j_{\beta}: Y_{\beta} \backslash Z_{\beta} \rightarrow Y_{\beta}$ denotes a natural inclusion), because $Z_{\beta}$ and $Y_{\beta}$ are smooth on a neighborhood of $Z_{\beta}$. So the assertion is reduced to the vanishing of (2.8.3) for $j=m+1$ by the distinguished triangle (2.8.2) because it gives the cokernel of can.

Using a one-parameter deformation $t \Psi(t \in \mathbb{C})$, we can reduce the assertion to the case $\Psi=0$. So we may assume that $Y_{B, \beta}$ is the intersection of $B$ with an affine cone $Y_{\beta}$ of a nonsingular conic, and $Z_{B, \beta}$ is its intersection with a linear space passing near the origin. Then we may omit the subscript $B$ by replacing $B$ with arbitrary large open balls and taking the limit. So the assertion follows from Artin's 
generalization of the weak Lefschetz theorem [1]. Indeed, the closure $\bar{Z}_{\beta}$ of $Z_{\beta}$ in the projective space intersects transversely the divisor at infinity $\bar{Y}_{\beta} \backslash Y_{\beta}$ of $Y_{\beta}$ so that we can first take the direct image with compact supports by $Y_{\beta} \backslash Z_{\beta} \rightarrow \bar{Y}_{\beta} \backslash \bar{Z}_{\beta}$, and then the usual direct image by $\bar{j}_{\beta}: \bar{Y}_{\beta} \backslash \bar{Z}_{\beta} \rightarrow \bar{Y}_{\beta}$. Thus the assertion is proved for $m$ odd.

In the case $m$ is even, let $D$ be a sufficiently small neighborhood of $\alpha$ in $\Delta_{c}$. For a variety $V$ of pure dimension $r$, let $\widetilde{H}_{c}^{j}(V)=H_{c}^{j}(V)$ for $j \neq 2 r$, and define $\widetilde{H}_{c}^{2 r}(V)$ to be the kernel of $\operatorname{Tr}: H_{c}^{2 r}(V) \rightarrow \mathbb{Q}(-r)$ which is induced by the canonical morphism of $\mathbb{Q}_{V}(r)[2 r]$ to the dualizing complex $\mathbb{D}_{V}$. Then $\widetilde{H}_{c}^{j}\left(Z_{B, \alpha}\right)=\mathbb{Q}$ for $j=m-1, m$ by calculating the cohomology of $\partial Z_{B, \alpha}$ and using the long exact sequence $\rightarrow H^{j-1}\left(\partial Z_{B, \alpha}\right) \rightarrow H_{c}^{j}\left(Z_{B, \alpha}\right) \rightarrow H^{j}\left(Z_{B, \alpha}\right) \rightarrow$. In particular, (2.7.4) for $\lambda=\alpha$ is not exact, and is extended to a long exact sequence. So we have to consider a distinguished triangle in the derived category of sheaves on $D$, which is defined by using the direct images with compact supports under the morphisms of $Z_{B, D}, Y_{B, D} \backslash$ $Z_{B, D}, Y_{B, D}$ to $D$ (in particular, the base change holds). They contain some shifted constant sheaves which are annihilated by taking the reduced cohomology in (2.7.2). So deleting them, we get a short exact sequence of shifted perverse sheaves as in (2.8.1) such that the stalk of the 0-th cohomology sheaf is given by (2.7.4). By the above calculation, we have $\mathcal{G}^{\prime}=\mathbf{R}\left(j_{D}\right)_{*} \mathbb{Q}_{D^{*}}$ and $\mathcal{G}^{\prime \prime}=\mathbb{Q}_{D}$ in this case.

Using an argument similar to the case $m$ odd, we see that var $: \varphi_{t} \mathcal{G} \rightarrow \psi_{t} \mathcal{G}$ is injective (reducing to the assertion for $\mathcal{G}^{\prime}$ in this case) where $t$ is a coordinate around $\alpha$. So it is enough to show the nontriviality of can $: \psi_{t} \mathcal{G} \rightarrow \varphi_{t} \mathcal{G}$. By the triangle (2.8.2) this is equivalent to $H_{c}^{m}\left(Y_{B, \alpha} \backslash Z_{B, \alpha}\right)=\mathbb{Q}$, because it gives the kernel of can. By duality it is further equivalent to

$$
H^{m}\left(Y_{B, \alpha} \backslash Z_{B, \alpha}\right)=\mathbb{Q} \text {. }
$$

By the same argument as above, we may omit the subscript $B$ by assuming that $Y_{\alpha}$ is the restriction of a nonsingular conic $\bar{Y}_{\alpha}$ in $\mathbb{P}^{m+1}$ to the affine space $\mathbb{A}^{m+1}, Z_{\alpha}$ is its intersection with a hyperplane $H$, which is an affine cone of a nonsingular conic in $\mathbb{P}^{m-1}=H \cap \mathbb{P}^{m}$, and the divisor at infinity $\partial Y_{\alpha}=\bar{Y}_{\alpha} \backslash Y_{\alpha}$ of $Y_{\alpha}$ is smooth. Then, using a projection from the vertex of the affine cone $Z_{\alpha}$, we see that $Y_{\alpha} \backslash Z_{\alpha}$ is isomorphic to the complement of the union of $\partial Y_{\alpha}$ and $H \cap \mathbb{P}^{m}$ in $\mathbb{P}^{m}=\mathbb{P}^{m+1} \backslash \mathbb{A}^{m+1}$. So (2.8.4) follows considering the long exact sequence containing the Gysin morphism by $\partial Y_{\alpha} \backslash H \rightarrow \mathbb{A}^{m}=\mathbb{P}^{m} \backslash H$. This completes the proofs of Lemma (2.8) and Proposition (2.7).

\section{Hodge-Theoretic Method}

In this section, we prove the nonvanishing of certain extension classes when the assumption on a differential form in Theorem (0.4) is satisfied.

3.1. Extension groups. With the notation and the assumptions of (2.1), we assume in this section that $f, g$ are smooth (by restricting $S$ ), and $\mathcal{F}^{\prime}, \mathcal{F}^{\prime \prime}$ are 
nonzero. We will consider whether (2.1.2) splits in the category of local systems. Let

$$
\mathcal{H}=\mathcal{H o m}\left(\mathcal{F}^{\prime \prime}, \mathcal{F}^{\prime}\right) .
$$

Then there is a canonical isomorphism

$$
\operatorname{Ext}^{1}\left(\mathbb{Q}_{S}, \mathcal{H}\right)=\operatorname{Ext}^{1}\left(\mathcal{F}^{\prime \prime}, \mathcal{F}^{\prime}\right)
$$

where the extension group is taken in the category of admissible variations of mixed Hodge structures [25] (or equivalently, in that of mixed Hodge modules [22]). These groups are identified (using [2]) with the scalar extension of the group of admissible normal functions, which are sections of the family of Jacobians $\left\{J\left(\mathcal{H}_{s}\right)\right\}_{s \in S}$ satisfying some good conditions [23]. (Here $\mathcal{H}$ also denotes a variation of mixed $\mathbb{Z}$-variation of Hodge structure whose scalar extension is $\mathcal{H}$ ). We have furthermore a short exact sequence

$$
0 \rightarrow \operatorname{Ext}^{1}\left(\mathbb{Q}, H^{0}(S, \mathcal{H})\right) \rightarrow \operatorname{Ext}^{1}\left(\mathbb{Q}_{S}, \mathcal{H}\right) \stackrel{r}{\rightarrow} \operatorname{Hom}\left(\mathbb{Q}, H^{1}(S, \mathcal{H})\right) \rightarrow 0,
$$

see [22], [29], where Ext and Hom are taken in the category of mixed Hodge structures or that of admissible variations of mixed Hodge structures. (Using [22], this follows from the adjoint relation between the direct image and the pull-back of mixed Hodge modules by $S \rightarrow p t$. Using [29], we get the above short exact sequence with the cohomology in the last term replaced by the intersection cohomology, which is a subgroup of the cohomology in this case. But this is enough for our purpose, although we can show that these two give the same by taking Hom, see also [8].)

Let $\mathcal{F}_{\mathbb{Q}}, \mathcal{H}_{\mathbb{Q}}$, etc. denote the underlying local systems. Then we have

$$
\operatorname{Ext}^{1}\left(\mathcal{F}_{\mathbb{Q}}^{\prime \prime}, \mathcal{F}_{\mathbb{Q}}^{\prime}\right)=H^{1}\left(S, \mathcal{H}_{\mathbb{Q}}\right)
$$

This is compatible with the last morphism $r$ in (3.1.2). Let $e$ be the extension class defined by the short exact sequence (2.1.2). Then (2.1.2) splits in the category of local systems if and only if $r(e)=0$.

3.2. Proposition (Non Lefschetz pencil case). With the notation and the assumptions of (2.1), assume $Y=Y_{0} \times S, f=\mathrm{pr}_{2}, S$ is an affine rational curve, and $\operatorname{pr}_{1} \circ i: X \rightarrow Y_{0}$ is birational, where $\operatorname{pr}_{i}$ is the $i$-th projection. Assume furthermore that $Y_{0}$ has a nontrivial differential form of the highest order. Then (2.1.2) does not split in the category of local systems. Furthermore, if there is a direct sum decomposition of variations of Hodge structures $\mathcal{F}^{\prime}=\mathcal{F}_{1}^{\prime} \oplus \mathcal{F}_{2}^{\prime}$ such that $\mathcal{F}_{1}^{\prime}$ is a constant variation, then the extension class between $\mathcal{F}^{\prime \prime}$ and $\mathcal{F}_{2}^{\prime}$ does not vanish in the category of local systems.

Proof. Consider the short exact sequence

$$
\mathrm{Gr}_{m}^{W} H^{m}(Y, X ; \mathbb{Q}) \stackrel{\alpha}{\rightarrow} \operatorname{Gr}_{m}^{W} H^{m}(Y, \mathbb{Q}) \stackrel{\beta}{\rightarrow} \operatorname{Gr}_{m}^{W} H^{m}(X, \mathbb{Q})
$$

where $W$ is the weight filtration of mixed Hodge structure [4]. Since $S$ is a smooth affine rational curve, $H^{1}(S, \mathbb{Q})$ has weights $>1$, and we have by the Künneth decomposition

$$
\operatorname{Gr}_{m}^{W} H^{m}(Y, \mathbb{Q})=H^{m}\left(Y_{0}, \mathbb{Q}\right) .
$$


Then the last morphism $\beta$ in (3.2.1) is identified with the restriction morphism

$$
H^{m}\left(Y_{0}, \mathbb{Q}\right) \rightarrow \operatorname{Gr}_{m}^{W} H^{m}(X, \mathbb{Q})
$$

by $X \rightarrow Y_{0}$, and its kernel is annihilated by the restriction morphism to a nonempty open subvariety of $Y_{0}$, because $X \rightarrow Y_{0}$ is birational.

Thus the kernel of $\beta$ has level $<m$ (where the level of a Hodge structure is the difference between the maximal and minimal numbers $p$ such that the $p$-th graded piece of the Hodge filtration does not vanish, see [4]). This implies that $\operatorname{Im} \beta \neq 0$, because $H^{m}\left(Y_{0}, \mathbb{Q}\right)$ has level $m$ by the hypothesis on the highest form. So we have a nonzero element

$$
u \in \operatorname{Gr}_{m}^{W} H^{m}(Y, \mathbb{Q})=H^{0}\left(S, R^{m} f_{*} \mathbb{Q}_{Y}\right)
$$

such that $\beta(u) \neq 0$. Using the semisimplicity of polarizable Hodge structures [4], we may assume that $\gamma_{m}(u)=0$ in $H^{0}\left(S, R^{m} g_{*} \mathbb{Q}_{X}\right)$ (or equivalently, in $H^{m}\left(X_{s}, \mathbb{Q}\right.$ ) for $s \in S)$, because $H^{m}\left(X_{s}, \mathbb{Q}\right)$ has level $<m$. Thus $u$ belongs to $H^{0}\left(S, \mathcal{F}^{\prime \prime}\right)$.

For the first morphism $\alpha$ in (3.2.1), consider the Leray spectral sequence in the category of mixed Hodge structures

$$
E_{2}^{p, q}=H^{p}\left(S, R^{q} h_{!} \mathbb{Q}_{U}\right) \Rightarrow H^{p+q}(Y, X ; \mathbb{Q}) .
$$

This degenerates at $E_{2}$, because $E_{2}^{p, q}=0$ unless $p=0$ or 1 . So we get the surjection

$$
\left.\mathrm{Gr}_{m}^{W} H^{m}(Y, X ; \mathbb{Q}) \rightarrow \operatorname{Gr}_{m}^{W} H^{0}(S, \mathcal{F}) \text { (see }(2.1) \text { for } \mathcal{F}\right) .
$$

Now assume that (2.1.2) splits in the category of local systems. Then there exists $v \in H^{0}(S, \mathcal{F})$ whose image in $H^{0}\left(S, \mathcal{F}^{\prime \prime}\right)$ is $u$. Here we may assume $v \in$ $\mathrm{Gr}_{m}^{W} H^{0}(S, \mathcal{F})$, because $H^{0}\left(S, \mathcal{F}^{\prime \prime}\right)$ is pure of weight $m$, and the image commutes with $\mathrm{Gr}_{m}^{W}$. But this contradicts the nonvanishing of $\beta(u)$ using the surjectivity of (3.2.3). So the first assertion follows.

For the last assertion, it is enough to show the vanishing of the extension class between $\mathcal{F}^{\prime \prime}$ and $\mathcal{F}_{1}^{\prime}$ as local systems. But this follows from (3.1.2) and (3.1.3) because the first cohomology of a constant variation of Hodge structures of weight -1 on a rational curve has weight $>0$ (if it is nonzero). This completes the proof of Proposition (3.2).

3.3. Proposition (Surface case). With the notation and the assumptions of (2.1), assume $m=1$ (i.e. $Y$ is a surface and $X$ is a curve), $S$ is an affine rational curve, and the fiber $Y_{s}$ of $f$ is not a rational curve. Assume further that $f: Y \rightarrow S$ can be extended to a proper smooth morphism $f^{\prime}: Y^{\prime} \rightarrow S^{\prime}$ so that $Y, S$ are open subvarieties of $Y^{\prime}$, $S^{\prime}$ respectively, and the closure $X^{\prime}$ of $X$ in $Y^{\prime}$ is nonsingular, but it is not smooth over $S^{\prime}$. Then (2.1.2) does not split in the category of local systems.

Proof. In this case we have

$$
\mathcal{F}_{s}^{\prime}=\widetilde{H}^{0}\left(X_{s}, \mathbb{Q}\right), \quad \mathcal{F}_{s}^{\prime \prime}=H^{1}\left(Y_{s}, \mathbb{Q}\right) .
$$

Then in the notation of (3.1.3) we have

$$
H^{0}(S, \mathcal{H})=\operatorname{Hom}\left(\mathcal{F}_{\mathbb{Q}}^{\prime \prime}, \mathcal{F}_{\mathbb{Q}}^{\prime}\right)=0,
$$


considering the monodromy of $\mathcal{F}_{s}^{\prime}$ around $S^{\prime} \backslash S$. So by (3.1.2) it is enough to show that the corresponding normal function in (3.1.1) is nontorsion, and the assertion is local on $S$ in the classical topology.

Let $s_{0} \in S^{\prime} \backslash S$, and $\Delta$ be an open disk around $s_{0}$ in $S^{\prime}$ such that $\Delta \cap S=$ $\Delta^{*}\left(:=\Delta \backslash\left\{s_{0}\right\}\right)$. Then the assumption on $S^{\prime}$ implies that there exist continuously $\Lambda_{s}=\left\{x_{s}, y_{s}\right\} \subset X_{s}$ for $s \in \Delta^{*}$ such that the action of the monodromy around $s_{0}$ on $\Lambda_{s}$ is nontrivial (replacing $s_{0}$ if necessary). Locally on $\Delta^{*}$, the difference $\left[x_{s}\right]-\left[y_{s}\right]$ defines an element $u_{s} \in \widetilde{H}^{0}\left(X_{s}\right)$ and also a point $\xi_{s}$ of the Jacobian $J\left(Y_{s}\right)$ of $Y_{s}$. Note that $\xi_{s}$ corresponds to the pull-back of the dual of the short exact sequence of mixed Hodge structures $(2.1 .2)_{s}$ by $u_{s}$ (using [2]), where $(2.1 .2)_{s}$ is the stalk at $s$ of the exact sequence (2.1.2).

We have $\xi_{s} \neq 0$ in $J\left(Y_{s}\right)$, because $Y_{s}$ is nonrational. If a nonzero multiple of $\xi_{s}$ vanishes locally on $\Delta^{*}$, it defines a locally constant section of the division points of the family of Jacobians over $\Delta$ (because $f$ is proper smooth over $\Delta$ ), and we get a contradiction by considering the monodromy around $s_{0}$. So the assertion follows.

3.4. Complement on the assumption of (3.3). The last assumption of Proposition (3.3) is satisfied in the case of a generic Lefschetz pencil as follows. Let $Y$ be a smooth surface embedded in a projective space $\mathcal{P}:=\mathbb{P}_{\mathbb{C}}^{r}(r>2)$. Let $X$ be a (locally closed) smooth curve on $Y$. Then there is a hyperplane of $\mathcal{P}$ which intersects $Y$ transversely, but is tangent to $X$.

Indeed, let $\mathcal{P}^{*}$ denote the dual projective space of $\mathcal{P}$ consisting of hyperplanes $H$ of $\mathcal{P}$, and $D_{Y}, D_{X}$ be the set of hyperplanes tangent to $Y, X$ respectively. By definition, $D_{Y}$ is the image of a $\mathbb{P}^{r-3}$-bundles $P_{Y}$ over $Y$ (where $P_{Y, y}$ consists of the hyperplanes tangent to $Y$ at $y$ ), and similarly for $X$ with $\mathbb{P}^{r-3}$ replaced by $\mathbb{P}^{r-2}$. Let $x \in X$, and assume $P_{X, x} \subset D_{Y}$. Then there exist a pencil of hyperplanes $\left\{H_{t}\right\}_{t \in \mathbb{P}^{1}}$ contained in $P_{X, x}$ and a smooth analytic curve $C$ locally defined on $Y$ together with a nonconstant holomorphic map $\rho: C \rightarrow \mathbb{P}^{1}$ such that $H_{\rho(c)}$ is tangent to $Y$ (and hence to $C$ ) at any $c \in C$. But this implies the constancy of $\rho$, which is a contradiction.

\section{Degeneration and Nearby Cycles}

In this section, we calculate the weight filtration of Steenbrink, and prove Theorem (0.4) using the results in Sections 2 and 3.

4.1. Family of hypersurfaces. With the notation of (1.1), assume condition (0.1) in Introduction. Let $V_{1}, V_{2}, V_{3}$ be vector subspaces of $\mathcal{A}_{Z}^{\delta}, \mathcal{A}^{d-\delta}, \mathcal{A}_{Z}^{d}$ such that $V_{1}, V_{3}$ generate $\mathcal{I}_{Z} \otimes \mathcal{O}_{Y}(\delta), \mathcal{I}_{Z} \otimes \mathcal{O}_{Y}(d)$ respectively and $V_{2}$ gives an embedding of $Y$ into a projective space. We assume $V_{3}$ is the image of $V_{3}^{\prime} \otimes V_{3}^{\prime \prime}$ where $V_{3}^{\prime}$ is a vector subspace of $\mathcal{A}_{Z}^{\delta}$ generating $\mathcal{O}_{Y}(\delta)$ outside $Z$ and $V_{3}^{\prime \prime}$ is a vector subspace of $\mathcal{A}^{d-\delta}$ giving an embedding of $Y$ into a projective space. In the proof of Theorem (0.4), $V_{1}, V_{3}^{\prime}$ and $V_{2}, V_{3}^{\prime \prime}$ will be respectively the restrictions of $\mathcal{A}_{Z^{\prime}}^{\delta}$ and $\mathcal{A}^{d-\delta}$ defined for some smooth projective variety $Y^{\prime}$ containing $Y$ where $Z^{\prime} \subset Y^{\prime}$ is a subvariety whose intersection with $Y$ is $Z$. (Actually, it is possible that $Z$ is empty, but $Z^{\prime}$ is not.) These are necessary to carry out an inductive argument in (4.7). 
We will identify $V_{1}, V_{2}, V_{3}$ with the corresponding affine spaces. Let $\widetilde{V}_{1}, \widetilde{V}_{2}, \widetilde{V}_{3}$ be their intersections with $\mathcal{V}_{Z}^{\delta}, \mathcal{V}^{d-\delta}, \mathcal{V}_{Z}^{d}$. Put $X_{P, Q}=X_{P} \cap X_{Q}, X_{P, Q, R}=X_{P} \cap X_{Q} \cap X_{R}$ for $P \in \mathcal{A}^{i}$, etc. Let

$$
S_{0}=\left\{(P, Q, R) \in \widetilde{V}_{1} \times \widetilde{V}_{2} \times \widetilde{V}_{3}: X_{P, Q}, X_{Q, R}, X_{P, Q, R} \text { are SCI }\right\},
$$

where SCI means smooth complete intersection. By [19] (or (1.2)), $S_{0}$ is non empty, and $X_{P, R}$ has at most isolated singularities, see also [13]. Here we can replace $S_{0}$ with a non empty subvariety because of (4.2) below. Let

$$
S^{\prime}=\left\{(P, Q, R, t) \in S_{0} \times \mathbb{C}^{*}: X_{P Q+t R} \text { is smooth }\right\},
$$

and $S^{\prime \prime}=\mathcal{V}_{Z}^{d} / \mathbb{C}^{*}$. We have a local system $L$ on $S^{\prime \prime}$ whose stalks are given by $H^{m}(X, \mathbb{Q})_{\perp Z}^{\operatorname{van}}$ in Conjecture $(0.2)$. Consider a morphism $\rho: S^{\prime} \rightarrow S^{\prime \prime}$ which associates $P Q+t R$ to $(P, Q, R, t)$. We apply the reduction argument in (4.2) below to this so that the proof of Theorem (0.4) is reduced to the pull-back of the local system to $S^{\prime}$. Here it is enough to show the indecomposability of $L$, because $L$ is semisimple by Deligne [4].

4.2. Reduction argument. Let $L$ be a local system on a connected analytic space $S^{\prime \prime}$. Then it is simple (resp. indecomposable) if there exists a morphism $\rho: S^{\prime} \rightarrow S^{\prime \prime}$ such that $\rho^{*} L$ is simple (resp. indecomposable). This follows from the fact that the functor $\rho^{*}$ is exact and faithful (or using the corresponding representation of the fundamental group). A similar assertion also holds for the nearby cycle functor $\psi$.

4.3. Semistable degeneration. With the notation of (4.1), there is a smooth projective morphism $f^{\prime}: \mathcal{X}^{\prime} \rightarrow S^{\prime}$ whose fiber $\mathcal{X}_{s}:=f^{\prime-1}(s)$ is $X_{P Q+t R}$ for $s=$ $(P, Q, R, t) \in S^{\prime}$. Let $L^{\prime}$ be a subsheaf of $R^{m} f_{*}^{\prime} \mathbb{Q}_{\mathcal{X}^{\prime}}$ whose stalk is the orthogonal complement of the subspace generated by $H^{m}(Y, \mathbb{Q})$ together with the cycle classes of the irreducible components of the closed subvariety $Z$. Then $L^{\prime}=\rho^{*} L$. Let

$$
S=\left\{(P, Q, R, t) \in S_{0} \times \mathbb{C}: X_{P Q+t R} \text { is smooth with } t \neq 0 \text { or } t=0\right\},
$$

i.e. $S$ is the disjoint union of $S^{\prime}$ and $S_{0}$. Then $f^{\prime}$ is naturally extended to $f: \mathcal{X} \rightarrow S$. However, $\mathcal{X}$ has certain singularities.

Let $\mathcal{C}$ be the closed subvariety of $\mathcal{X}$ whose fiber over $(P, Q, R, t) \in S$ is $X_{Q, R}$. Note that its restriction over $S^{\prime}$ is a locally principal divisor. Let $\pi: \widetilde{\mathcal{X}} \rightarrow \mathcal{X}$ be the blow-up along $\mathcal{C}$. Let $\widetilde{f}: \widetilde{\mathcal{X}} \rightarrow S$ denote the composition with $f$. We also denote by $t$ the function defined by the last component of $(P, Q, R, t)$. Let $\widetilde{\mathcal{X}}_{0}, \mathcal{X}_{0}, S_{0}$ be the subvarieties of $\widetilde{\mathcal{X}}, \mathcal{X}, S$ defined by $t=0$ (this is compatible with the previous definition of $S_{0}$ ). We have the induced morphism $\tilde{f}_{0}: \widetilde{\mathcal{X}}_{0} \rightarrow S_{0}$.

Let $\mathcal{X}_{P}$ be the closed subvariety of $\mathcal{X}$ whose fiber over $(P, Q, R) \in S_{0}$ is $X_{P}$ (using the projection $\mathcal{X} \rightarrow S_{0}$ ), and similarly for $\mathcal{X}_{P, Q}, \mathcal{X}_{P, Q, R}$, etc. (Note that $\mathcal{X}_{P}, \mathcal{X}_{Q}$ are contained in $\mathcal{X}_{0}$.) Let $\mathcal{X}_{P}^{\sim}$ be the blow-up of $\mathcal{X}_{P}$ along $\mathcal{X}_{P, Q, R}$. Then $\widetilde{\mathcal{X}}_{0}$ is a divisor with normal crossings on a smooth variety $\widetilde{\mathcal{X}}$, and its irreducible components are $\mathcal{X}_{P}^{\sim}$ and $\mathcal{X}_{Q}$, see [10].

Let $\psi$ denotes the nearby cycle functor, see [5]. Then

$$
R^{m} \tilde{f}_{*} \psi_{t} \mathbb{Q}_{\tilde{\mathcal{X}}}=\psi_{t} R^{m} \tilde{f}_{*} \mathbb{Q}_{\tilde{\mathcal{X}}}\left(=\psi_{t} R^{m} f_{*}^{\prime} \mathbb{Q}_{\mathcal{X}^{\prime}}\right),
$$


because the nearby cycle functor commutes with the direct image under a proper morphism.

Let $W$ be the weight filtration on $\psi_{t} \mathbb{Q}_{\tilde{\mathcal{X}}}$. By Steenbrink [24], we have

$$
\begin{aligned}
\operatorname{Gr}_{m-1}^{W} \psi_{t} \mathbb{Q}_{\tilde{\mathcal{X}}} & =\mathbb{Q}_{\mathcal{X}_{P, Q}}[-1], \\
\operatorname{Gr}_{m}^{W} \psi_{t} \mathbb{Q}_{\widetilde{\mathcal{X}}} & =\mathbb{Q}_{\mathcal{X}_{Q}} \oplus \mathbb{Q}_{\mathcal{X}}, \\
\operatorname{Gr}_{m+1}^{W} \psi_{t} \mathbb{Q}_{\tilde{\mathcal{X}}} & =\mathbb{Q}_{\mathcal{X}_{P, Q}}(-1)[-1],
\end{aligned}
$$

and $\operatorname{Gr}_{k}^{W} \psi_{t} \mathbb{Q}_{\tilde{\mathcal{X}}}=0$ for $|k-m|>1$.

4.4. Weight spectral sequence. Consider the weight spectral sequence in the category of local systems

$$
E_{1}^{-k, j+k}=R^{j} \tilde{f}_{*} \operatorname{Gr}_{k}^{W} \psi_{t} \mathbb{Q}_{\widetilde{\mathcal{X}}} \Rightarrow \psi_{t} R^{j} f_{*}^{\prime} \mathbb{Q}_{\mathcal{X}^{\prime}}
$$

This degenerates at $E_{2}$ by [24]. (In this case, it also follows from an easy calculation.) The local system $\left\{H^{j}\left(X_{P}\right)\right\}$ on $S_{0}$ can be identified with $R^{j} \widetilde{f}_{*} \mathbb{Q}_{\mathcal{X}_{P}}$, and similarly for $\left\{H^{j}\left(X_{P}^{\sim}\right)\right\}$, etc., where the cohomology groups are with rational coefficients unless otherwise stated explicitly. We have

$$
H^{j}\left(X_{P}^{\sim}\right)=H^{j}\left(X_{P}\right) \oplus H^{j-2}\left(X_{P, Q, R}\right)(-1),
$$

because

$$
\mathbf{R} \pi_{*} \mathbb{Q}_{\mathcal{X}_{P}^{\sim}}=\mathbb{Q} \mathcal{X}_{P} \oplus \mathbb{Q} \mathcal{X}_{P, Q, R}(-1)[-2],
$$

by the decomposition theorem [1]. Let $\mathcal{Z}_{P}^{\widetilde{P}}=\pi^{-1}\left(X_{P, Q, R}\right)$. Then a canonical morphism $\mathbb{Q}_{\mathcal{X}_{P, Q, R}}(-1)[-2] \rightarrow \mathbf{R} \pi_{*} \mathbb{Q}_{\mathcal{X}_{\tilde{P}}}$ and its right inverse (up to a sign) are given by the compositions

$$
\begin{aligned}
& \mathbb{Q}_{\mathcal{X}_{P, Q}, R}(-1)[-2] \rightarrow \mathbf{R} \pi_{*} \mathbb{Q}_{\mathcal{Z}_{\widetilde{P}}}(-1)[-2] \rightarrow \mathbf{R} \pi_{*} \mathbb{Q}_{\mathcal{X}_{P}}, \\
& \mathbf{R} \pi_{*} \mathbb{Q}_{\mathcal{X}_{\tilde{P}}} \rightarrow \mathbf{R} \pi_{*} \mathbb{Q}_{\mathcal{Z}_{\tilde{P}}} \rightarrow \mathbb{Q}_{\mathcal{X}_{P, Q}, R}(-1)[-2] .
\end{aligned}
$$

The differential $d_{1}$ of the spectral sequence is induced by the extension classes between the $\mathrm{Gr}_{k}^{W}$ which are given by the restriction and Gysin morphisms. Indeed, the extension class between $\mathrm{Gr}_{m-1}^{W}$ and $\mathrm{Gr}_{m}^{W}$ corresponds to a morphism $\mathrm{Gr}_{m}^{W} \rightarrow$ $\mathrm{Gr}_{m-1}^{W}[1]$ in the derived category of $\mathbb{Q}$-modules on $\widetilde{\mathcal{X}}$ (see [26]) and it is given by the restriction morphisms

$$
\mathbb{Q}_{\mathcal{X}_{Q}} \rightarrow \mathbb{Q}_{\mathcal{X}_{P, Q}}, \quad \mathbb{Q}_{\mathcal{X}_{\tilde{P}}} \rightarrow \mathbb{Q}_{\mathcal{X}_{P, Q}}
$$

The direct image by $\pi$ of the last morphism is given by the restriction and Gysin morphisms

$$
\mathbb{Q}_{\mathcal{X}_{P}} \rightarrow \mathbb{Q}_{\mathcal{X}_{P, Q}}, \quad \mathbb{Q}_{\mathcal{X}_{P, Q, R}}(-1)[-2] \rightarrow \mathbb{Q}_{\mathcal{X}_{P, Q}}
$$

(up to signs), using (4.4.2). We have a similar assertion between $\mathrm{Gr}_{m}^{W}$ and $\mathrm{Gr}_{m+1}^{W}$.

Let $H^{m-1}\left(X_{P, Q}\right)^{\text {van }}$ denote the orthogonal complement of the image of the (injective) restriction morphism $H^{m-1}(Y) \rightarrow H^{m-1}\left(X_{P, Q}\right)$ where $H^{m-1}(Y)$ can be replaced with $H^{m-1}\left(X_{P}\right)$ by the weak Lefschetz theorem, and similarly for $H^{m-2}\left(X_{P, Q, R}\right)^{\text {van }}$ 
and also for $H^{m}\left(X_{P}\right)^{\text {van }}$. Then we have the direct sum decompositions

$$
\begin{aligned}
& H^{m}\left(X_{P}\right)=H^{m}\left(X_{P}\right)^{\operatorname{van}} \oplus H^{m}(Y)^{\operatorname{prim}} \oplus H^{m-2}(Y)(-1), \\
& H^{m}\left(X_{Q}\right)=H^{m}\left(X_{Q}\right)^{\operatorname{van}} \oplus H^{m}(Y)^{\operatorname{prim}} \oplus H^{m-2}(Y)(-1), \\
& H^{m-2}\left(X_{P, Q, R}\right)=H^{m-2}\left(X_{P, Q, R}\right)^{\operatorname{van}} \oplus H^{m-2}(Y),
\end{aligned}
$$

together with the isomorphisms

$$
H^{m-2}\left(X_{P, Q}\right)(-1)=H^{m}\left(X_{P, Q}\right)=H^{m-2}(Y)(-1) .
$$

Here $H^{m}(Y)^{\text {prim }}$ denotes the primitive cohomology, and $H^{m-2}(Y)(-1)$ in the first two decompositions of (4.4.4) is actually the image of $H^{m-2}(Y)(-1)$ by the action of the cohomology class of the hyperplane section.

We see that the stalk of $\operatorname{Gr}_{m}^{W} \psi_{t} R^{m} f_{*}^{\prime} \mathbb{Q} \mathcal{X}^{\prime}$ is the cohomology of the complex

$$
\begin{aligned}
H^{m-2}\left(X_{P, Q}\right)(-1) \rightarrow H^{m}\left(X_{P}\right) \oplus H^{m}\left(X_{Q}\right) \oplus H^{m-2} & \left(X_{P, Q, R}\right)(-1) \\
& \rightarrow H^{m}\left(X_{P, Q}\right)
\end{aligned}
$$

where the morphisms are induced by the restriction and Gysin morphisms up to signs. Using (4.4.4), the first morphism of (4.4.5) is given by the identity on $H^{m-2}(Y)(-1)$ up to signs, and the last morphism is given by the multiplications on $H^{m-2}(Y)(-1)$ by $d-\delta, \delta, d$ up to signs. Indeed, the composition of the Gysin and restriction morphisms

$$
H^{m-2}\left(X_{P, Q}\right)(-1) \rightarrow H^{m}\left(X_{P}\right) \rightarrow H^{m}\left(X_{P, Q}\right)
$$

coincides with the action of the restriction of the cycle class of $X_{P, Q}$ in $X_{P}$.

Similarly we can verify

$$
\begin{aligned}
\mathrm{Gr}_{m-1}^{W} \psi_{t} R^{m} f_{*}^{\prime} \mathbb{Q}_{\mathcal{X}^{\prime}} & =\left\{H^{m-1}\left(X_{P, Q}\right)^{\operatorname{van}}\right\}, \\
\operatorname{Gr}_{m+1}^{W} \psi_{t} R^{m} f_{*}^{\prime} \mathbb{Q}_{\mathcal{X}^{\prime}} & =\left\{H^{m-1}\left(X_{P, Q}\right)^{\operatorname{van}}(-1)\right\} .
\end{aligned}
$$

Here the first assertion is easy, and the second follows from it using duality.

Note that the extension classes between the $\mathrm{Gr}_{k}^{W}$ are induced by the restriction and Gysin morphisms in the derived category of sheaves on $\widetilde{\mathcal{X}}$ as above.

4.5. Limit of the invariant part. Taking the nearby cycle functor (i.e. passing to the limit by $t \rightarrow 0)$, the restriction morphism $H^{m}(Y) \rightarrow H^{m}\left(X_{P Q+t R}\right)$ induces a morphism of $H^{m}(Y)$ to the middle term of the complex (4.4.5), which is defined (up to signs) by using the isomorphism

$$
H^{m}(Y)=H^{m}(Y)^{\operatorname{prim}} \oplus H^{m-2}(Y)(-1)
$$

together with (4.4.4). So $\mathrm{Gr}_{m}^{W}$ of the limit of $H^{m}\left(X_{P Q+t R}\right) / H^{m}(Y)$ is given by

$$
\left(H^{m}\left(X_{P}\right)^{\operatorname{prim}} \oplus H^{m}\left(X_{Q}\right)^{\operatorname{prim}} \oplus H^{m-2}\left(X_{P, Q, R}\right)^{\operatorname{van}}(-1)\right) / H^{m}(Y)^{\operatorname{prim}} .
$$

Note that

$$
H^{m}\left(X_{P}\right)^{\operatorname{prim}}=H^{m-2}\left(X_{P}\right)^{\operatorname{van}} \oplus H^{m}(Y)^{\text {prim }}
$$


( similarly for $H^{m}\left(X_{Q}\right)^{\text {prim }}$ ), and the division by $H^{m}(Y)^{\text {prim }}$ is defined by using the diagonal morphism. In particular, the quotient (4.5.2) is isomorphic to

$$
H^{m}\left(X_{P}\right)^{\operatorname{prim}} \oplus H^{m}\left(X_{Q}\right)^{\operatorname{van}} \oplus H^{m-2}\left(X_{P, Q, R}\right)^{\operatorname{van}}(-1),
$$

Here $\left\{H^{m}\left(X_{Q}\right)^{\operatorname{van}}\right\}$ is an irreducible local system on $S_{0}$. Assuming an appropriate inductive hypothesis (see (4.7) below), this also holds for the quotient of $H^{m-2}\left(X_{P, Q, R}\right)^{\text {van }}$ by the subspace generated by the cycle classes of the intersection with $X_{Q}$ of the $(m / 2)$-dimensional irreducible components of $Z$ if $m>2$.

4.6. Cycle classes of the irreducible components. The cycle classes of the irreducible components of $Z$ in the limit of $H^{m}\left(X_{P Q+t R}\right)$ are given by using

$$
H_{\mathrm{BM}}^{m-2}\left(X_{P, R}\right)(-1) \rightarrow H^{m}\left(X_{P}\right) \oplus H^{m-2}\left(X_{P, Q, R}\right)(-1),
$$

which is induced by the Gysin and restriction morphisms up to signs. (This can be verified by using (4.4.3).) Here $H_{\mathrm{BM}}^{j}(V)$ denotes Borel-Moore cohomology for an equidimensional variety $V$ in general, and is defined by $H^{j}\left(V, \mathbb{D}_{V}(-n)[-2 n]\right)$, where $\mathbb{D}_{V}$ is the dualizing complex and $n=\operatorname{dim} V$. If $V$ is compact, it is isomorphic to $H_{2 n-j}(V)(-n)$. The restriction of the dualizing complex to the smooth part $V_{\text {reg }}$ is isomorphic to $\mathbb{Q}_{V_{\text {reg }}}(n)[2 n]$, and we get the restriction morphism $H_{\mathrm{BM}}^{j}(V) \rightarrow H^{j}\left(V^{\prime}\right)$ for any subvariety $V^{\prime}$ of $V_{\text {reg. }}$.

The morphism to the second factor of (4.6.1) is injective, i.e. the Gysin morphism $H^{m-2}\left(X_{P, Q, R}\right)(-1) \rightarrow H^{m}\left(X_{P, R}\right)$ is surjective. This follows from Artin's theorem (see [1]) which asserts the vanishing of $H^{m}\left(X_{P, R} \backslash X_{P, Q, R}\right)$ because $X_{P, R} \backslash X_{P, Q, R}$ is affine and the constant sheaf on it is semi-perverse up to a shift of complex by $m-1$.

We can verify that the limit of the cycle class of $Z$ is then defined by using the cycle class of the cycle in $X_{P}$ and the cycle class in $X_{P, Q, R}$ of the intersection of the cycle with $X_{Q}$.

4.7. Proof of Theorem (0.4). Assume first $m=2$ (and $Y^{\prime}$ in (4.1) is $Y$ ). In this case, $X_{P, Q, R}$ has dimension 0 and is not connected. We first fix $P, R$ and consider $\left\{X_{P, Q}\right\}_{Q}$ and $\left\{X_{P, Q, R}\right\}_{Q}$. Let $Z_{i}(i>0)$ be the 1-dimensional irreducible components of $Z$. Then $X_{P, R}$ is the union of $\bigcup_{i>0} Z_{i}$ and an irreducible curve $Z_{0}$, because $X_{P, R} \backslash Z$ is smooth and connected by (1.2) (where $b=0$ if $\operatorname{dim} Z=1$ ), see also [13], [19].

We apply the dual of Proposition (2.7) or (3.3) to $\left\{X_{P, Q}\right\}_{Q}$ and $\left\{Z_{i, Q}\right\}_{Q}$ for $i \geq 0$, where $Z_{i, Q}=Z_{i} \cap X_{Q}$. Then we get the nontriviality of the extension class between $\operatorname{Gr}_{m-1}^{W}=\left\{H^{m-1}\left(X_{P, Q}\right)^{\text {van }}\right\}$ and $\left\{\widetilde{H}^{m-2}\left(Z_{i, Q}\right)(-1)\right\}$ for $i \geq 0$, using (4.2). Similarly we apply Proposition (2.7) or (3.2) to show the nontriviality of the extension class between $\operatorname{Gr}_{m-1}^{W}$ and $\left\{H^{m}\left(X_{Q}\right)^{\text {van }}\right\}$, where we fix $Q, R$ or $P, R$ to apply the propositions. We use Proposition (2.3) for the extension between $\mathrm{Gr}_{m-1}^{W}$ and any simple factor of $\left\{H^{m}\left(X_{P}\right)^{\text {prim }}\right\}$. (Note that $\left\{H^{m}\left(X_{P}\right)^{\text {prim }}\right\}$ is semisimple by $[4]$.)

Thus we get the nontriviality of the extension class between $\mathrm{Gr}_{m-1}^{W} \psi_{t} L^{\prime}$ and each simple factor of $\mathrm{Gr}_{m}^{W} \psi_{t} L^{\prime}$ in the notation of (4.3), because $\operatorname{Gr}_{m}^{W} \psi_{t} L^{\prime}$ is isomorphic to the quotient of (4.5.4) by the image of the cycle classes of the $Z_{i}(i>0)$ (where 
the last term vanishes unless $\operatorname{dim} Z=1$ ), see (4.6). We have the dual argument for the extension between $\operatorname{Gr}_{m}^{W}$ and $\operatorname{Gr}_{m+1}^{W}$. Note that the $\left\{\widetilde{H}^{m-2}\left(Z_{i, Q}\right)\right\}$ are not isomorphic to each other (considering the monodromy around $Q$ such that $X_{Q}$ is tangents to $Z_{j}$ at a smooth point for one $j$, but intersects $Z_{i}$ transversely at smooth points for $i \neq j)$. We also see that any simple factor of $\left\{H^{m}\left(X_{P}\right)^{\text {prim }}\right\}$ is not isomorphic to $\left\{\widetilde{H}^{m-2}\left(Z_{i, Q}\right)\right\}$, nor to $\left\{H^{m}\left(X_{Q}\right)^{\text {van }}\right\}$ (fixing $P$ ), and similarly between the last two.

Assume that there is a decomposition $\psi_{t} L^{\prime}=L_{1} \oplus L_{2}$ in the notation of (4.3). We may assume that the $L_{i}$ are stable by the action of the monodromy $T$, because the decomposition is induced by that of $L^{\prime}$. In our case the weight filtration $W$ is defined by $W^{m-1}=\operatorname{Im} N$ and $W^{m}=\operatorname{Ker} N$. Here $N=T-i d$ because $N^{2}=0$. If, for example, $L_{1}$ is not contained in $W^{m}$, then $N L_{1}=W^{m-1}$, because $N L_{1} \subset W^{m-1}$ is nonzero and $W^{m-1}=\mathrm{Gr}_{m-1}^{W}$ is simple. In this case $L_{2}$ is contained in $W^{m}$, because otherwise it also contains $W^{m-1}$. So the decomposition induces that of $\mathrm{Gr}_{m}^{W}$, and the nontriviality of the above extension classes implies the triviality of the decomposition. Thus, using Deligne's semisimplicity theorem, Theorem (0.4) is proved for $m=2$.

If $m=1$, then $X_{P}$ is smooth and connected by Theorem (1.2) with $r=1$, and $X_{P, Q, R}$ is empty. So the argument is essentially same as above.

Assume now $m>2$. We apply an inductive hypothesis to $X_{P, Q}$ and $Z \cap X_{Q}$ to show the irreducibility of $\left\{H^{m-2}\left(X_{P, Q, R}\right)^{\text {van }}\right\}_{R}$ divided by the image of the cycle classes of the irreducible components of $Z$, see (4.6). To carry out this induction we take general $P_{i}, Q_{i}, R_{i}$ such that $R_{i}=P_{i+1} Q_{i+1}+R_{i+1}$ and $Z$ is contained in $X_{P_{i}}, X_{R_{i}}$. Note that the singular locus of $\bigcap_{1 \leq j \leq i} X_{P_{j}}$ has dimension $\leq i-2$, see [19] (or (1.2)). We apply the above argument to the restriction of $\left(P_{i} \cdot Q_{i}, R_{i}\right)$ to $Y^{\prime \prime}:=\bigcap_{1 \leq j<i} X_{P_{j}, Q_{j}}$ for each $i$, and proceed by decreasing induction on $i$. This is allowed by the definition of $S_{0}$ in (4.1) (where $Y^{\prime}, Y$ in (4.1) is $Y, Y^{\prime \prime}$ here). Here we use Proposition (2.7) or (3.2) (instead of (3.3)) to show the nontriviality of the extension between $\operatorname{Gr}_{W}^{m-1}$ and $\left\{H^{m-2}\left(X_{P, Q, R}\right)^{\operatorname{van}}(-1)\right\}$ divided by the cycle classes as above. Note that even if $Y^{\prime \prime} \cap Z$ is empty, we still have some restriction to $\left(P_{i} . Q_{i}, R_{i}\right)$ coming from $Z$. At the first step of the induction, we have $m=2$ or 1 . This completes the proof of Theorem (0.4).

4.8. Generalization of Theorem (0.4). The assertion also holds for smooth zero loci of sections of $\mathcal{L}_{1} \otimes \mathcal{L}_{2}$ containing $Z$ where $\mathcal{L}_{1}, \mathcal{L}_{2}$ are line bundles such that $\mathcal{L}_{1} \otimes \mathcal{I}_{Z}$ is generated by its global sections, and $\mathcal{L}_{2}$ is very ample and satisfies one of the following two conditions: either a general smooth hyperplane section of $\mathcal{L}_{2}$ has a nontrivial differential form of the highest degree, or the 2-jets at each point is generated by the global sections of $\mathcal{L}_{2}$ (e.g. it is the $(d-\delta)$-ple tensor of a very ample line bundle with $d \geq \delta+2$ ).

\section{REFERENCES}

[1] A. Beilinson, J. Bernstein and P. Deligne, Faisceaux pervers, Astérisque, vol. 100, Soc. Math. France, Paris, 1982. 
[2] J. Carlson, Extensions of mixed Hodge structures, in Journées de Géométrie Algébrique d'Angers 1979, Sijthoff-Noordhoff Alphen a/d Rijn, 1980, pp. 107-128.

[3] H. Clemens, Degeneration of Kähler manifolds, Duke Math. J. 44 (1977), 215-290.

[4] P. Deligne, Théorie de Hodge I, Actes Congrès Intern. Math., 1970, vol. 1, 425-430; II, Publ. Math. IHES, 40 (1971), 5-57; III ibid., 44 (1974), 5-77.

[5] _ Le formalisme des cycles évanescents, in SGA7 XIII and XIV, Lect. Notes in Math. vol. 340, Springer, Berlin, 1973, pp. 82-115 and 116-164.

[6] _. La formule de Picard-Lefschetz, in SGA7 XV, Lect. Notes in Math. vol. 340, Springer, Berlin, 1973, pp. 165-197.

[7] A. Dimca, Sheaves in Topology, Universitext, Springer, Berlin (2003), to appear.

[8] A. Dimca and M. Saito, Monodromy at infinity and the weights of cohomology, Compos. Math. 138 (2003), 55-71.

[9] D. Eisenbud, Commutative algebra with a view toward algebraic geometry, Springer, New York, 1995.

[10] P. Griffiths and J. Harris, On the Noether-Lefschetz theorem and some remarks on codimension two cycles, Math. Ann. 271 (1985) 31-51.

[11] L. Illusie, Autour du théorème de monodromie locale, Astérisque 223 (1994), 9-57.

[12] N. Katz, Etude cohomologique des pinceaux de Lefschetz, in Lect. Notes in Math., vol. 340, Springer Berlin, 1973, pp. 254-327.

[13] S. Kleiman and A. Altman, Bertini theorems for hypersurface sections containing a subscheme, Comm. Algebra 7 (1979), 775-790.

[14] S. Lefschetz, L'analysis situs et la géométrie algébrique, Gauthier-Villars, Paris, 1924.

[15] J.D. Lewis, A survey of the Hodge conjecture (Second edition), Monograph Series 10, American Mathematical Society, Providence, RI, 1999.

[16] A.F. Lopez, Noether-Lefschetz theory and the Picard group of projective surfaces, Mem. Amer. Math. Soc. 89 (1991).

[17] J. Milnor, Singular points of complex hypersurfaces, Ann. Math. Stud. vol. 61, Princeton Univ. Press, 1968.

[18] A. Otwinowska, Composantes de petite codimension du lieu de Noether-Lefschetz; un argument en faveur de la conjecture de Hodge pour les hypersurfaces, J. Algebraic Geom. 12 (2003), 307-320.

[19] - Monodromie d'une famille d'hypersurfaces, preprint.

[20] _ Sur les variétés de Hodge des hypersurfaces, preprint (math.AG/0401092).

[21] M. Saito, Modules de Hodge polarisables, Publ. RIMS, Kyoto Univ., 24 (1988), 849-995.

[22] — Mixed Hodge Modules, Publ. RIMS, Kyoto Univ., 26 (1990), 221-333.

[23] - Admissible normal functions, J. Alg. Geom. 5 (1996), 235-276.

[24] J.H.M. Steenbrink, Limits of Hodge structures, Inv. Math. 31 (1975/76), 229-257.

[25] J.H.M. Steenbrink and S. Zucker, Variation of mixed Hodge structure I, Inv. Math. 80 (1985), $489-542$.

[26] J.-L. Verdier, Catégories dérivées, in SGA 4 1/2, Lect. Notes in Math., Springer, Berlin, vol. 569, 1977, pp. 262-311.

[27] _ Dualité dans la cohomologie des espaces localement compacts, Séminaire Bourbaki, No. 300.

[28] C. Voisin, Hodge theory and complex algebraic geometry, II, Cambridge University Press, Cambridge, 2003.

[29] S. Zucker, Hodge theory with degenerating coefficients, $L_{2}$-cohomology in the Poincaré metric, Ann. Math., 109 (1979), 415-476. 
Laboratoire de Mathématiques, Université Paris-Sud - BÂt 425, 91405 Orsay Cedex, France

E-mail address: Ania.Otwinowska@math.u-psud.fr

RIMS Kyoto University, Kyoto 606-8502, JapAN

E-mail address: msaito@kurims.kyoto-u.ac.jp 